\title{
Diffractive Deep-Inelastic Scattering with a Leading Proton at HERA
}

\author{
H1 Collaboration
}

\begin{abstract}
The cross section for the diffractive deep-inelastic scattering process $e p \rightarrow e X p$ is measured, with the leading final state proton detected in the H1 Forward Proton Spectrometer. The data analysed cover the range $x_{\mathbb{P}}<0.1$ in fractional proton longitudinal momentum loss, $0.08<|t|<0.5 \mathrm{GeV}^{-2}$ in squared four-momentum transfer at the proton vertex, $2<Q^{2}<50 \mathrm{GeV}^{2}$ in photon virtuality and $0.004<\beta=x / x_{\mathbb{P}}<1$, where $x$ is the Bjorken scaling variable. For $x_{\mathbb{P}} \lesssim 10^{-2}$, the differential cross section has a dependence of approximately $\mathrm{d} \sigma / \mathrm{d} t \propto e^{6 t}$, independently of $x_{\mathbb{P}}, \beta$ and $Q^{2}$ within uncertainties. The cross section is also measured triple differentially in $x_{\mathbb{P}}, \beta$ and $Q^{2}$. The $x_{\mathbb{P}}$ dependence is interpreted in terms of an effective pomeron trajectory with intercept $\alpha_{\mathbb{P}}(0)=1.114 \pm 0.018$ (stat.) \pm 0.012 (syst.) ${ }_{-0.020}^{+0.040}$ (model) and a sub-leading exchange. The data are in good agreement with an $\mathrm{H} 1$ measurement for which the event selection is based on a large gap in the rapidity distribution of the final state hadrons, after accounting for proton dissociation contributions in the latter. Within uncertainties, the dependence of the cross section on $x$ and $Q^{2}$ can thus be factorised from the dependences on all studied variables which characterise the proton vertex, for both the pomeron and the sub-leading exchange.
\end{abstract}




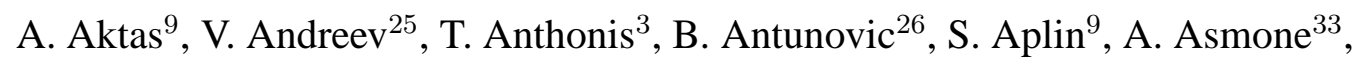

A. Astvatsatourov ${ }^{3}$, A. Babaev ${ }^{24, \dagger}$, S. Backovic ${ }^{30}$, A. Baghdasaryan $^{37}$, P. Baranov $^{25}$,

E. Barrelet ${ }^{29}$, W. Bartel ${ }^{9}$, S. Baudrand ${ }^{27}$, S. Baumgartner ${ }^{39}$, M. Beckingham ${ }^{9}$, O. Behnke ${ }^{12}$,

O. Behrendt ${ }^{6}$, A. Belousov ${ }^{25}$, N. Berger ${ }^{39}$, J.C. Bizot ${ }^{27}$, M.-O. Boenig ${ }^{6}$, V. Boudry ${ }^{28}$,

J. Bracinik $^{26}$, G. Brandt ${ }^{12}$, V. Brisson ${ }^{27}$, D. Bruncko ${ }^{15}$, F.W. Büsser ${ }^{10}$, A. Bunyatyan ${ }^{11,37}$,

G. Buschhorn ${ }^{26}$, L. Bystritskaya ${ }^{24}$, A.J. Campbell ${ }^{9}$, F. Cassol-Brunner ${ }^{21}$, K. Cerny ${ }^{32}$,

V. Cerny ${ }^{15,46}$, V. Chekelian ${ }^{26}$, J.G. Contreras ${ }^{22}$, J.A. Coughlan ${ }^{4}$, B.E. Cox ${ }^{20}$, G. Cozzika ${ }^{8}$,

J. Cvach ${ }^{31}$, J.B. Dainton ${ }^{17}$, W.D. Dau ${ }^{14}$, K. Daum ${ }^{36,42}$, Y. de Boer ${ }^{24}$, B. Delcourt ${ }^{27}$,

M. Del Degan ${ }^{39}$, A. De Roeck ${ }^{9,44}$, E.A. De Wolf ${ }^{3}$, C. Diaconu ${ }^{21}$, V. Dodonov ${ }^{11}$, A. Dubak ${ }^{30,45}$,

G. Eckerlin ${ }^{9}$, V. Efremenko ${ }^{24}$, S. Egli ${ }^{35}$, R. Eichler ${ }^{35}$, F. Eisele ${ }^{12}$, A. Eliseev ${ }^{25}$, E. Elsen ${ }^{9}$,

S. Essenov ${ }^{24}$, A. Falkewicz ${ }^{5}$, P.J.W. Faulkner ${ }^{2}$, L. Favart ${ }^{3}$, A. Fedotov ${ }^{24}$, R. Felst ${ }^{9}$, J. Feltesse ${ }^{8}$,

J. Ferencei ${ }^{15}$, L. Finke ${ }^{10}$, M. Fleischer ${ }^{9}$, G. Flucke ${ }^{33}$, A. Fomenko ${ }^{25}$, G. Franke ${ }^{9}$, T. Frisson ${ }^{28}$,

E. Gabathuler ${ }^{17}$, E. Garutti ${ }^{9}$, J. Gayler ${ }^{9}$, C. Gerlich ${ }^{12}$, S. Ghazaryan ${ }^{37}$, S. Ginzburgskaya ${ }^{24}$,

A. Glazov ${ }^{9}$, I. Glushkov ${ }^{38}$, L. Goerlich ${ }^{5}$, M. Goettlich ${ }^{9}$, N. Gogitidze ${ }^{25}$, S. Gorbounov ${ }^{38}$,

C. $\mathrm{Grab}^{39}$, T. Greenshaw ${ }^{17}$, M. Gregori ${ }^{18}$, B.R. Grell ${ }^{9}$, G. Grindhammer ${ }^{26}$, C. Gwilliam ${ }^{20}$,

D. Haidt ${ }^{9}$, M. Hansson ${ }^{19}$, G. Heinzelmann ${ }^{10}$, R.C.W. Henderson ${ }^{16}$, H. Henschel ${ }^{38}$,

G. Herrera ${ }^{23}$, M. Hildebrandt ${ }^{35}$, K.H. Hiller ${ }^{38}$, D. Hoffmann ${ }^{21}$, R. Horisberger ${ }^{35}$,

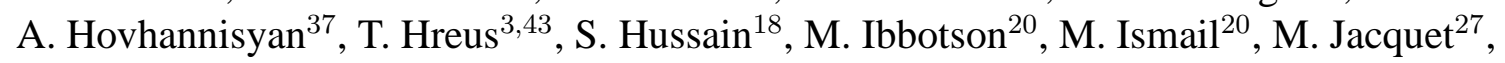

X. Janssen ${ }^{3}$, V. Jemanov ${ }^{10}$, L. Jönsson ${ }^{19}$, D.P. Johnson ${ }^{3}$, A.W. Jung ${ }^{13}$, H. Jung ${ }^{19,9}$,

M. Kapichine ${ }^{7}$, J. Katzy ${ }^{9}$, I.R. Kenyon ${ }^{2}$, C. Kiesling ${ }^{26}$, M. Klein ${ }^{38}$, C. Kleinwort ${ }^{9}$,

T. Klimkovich ${ }^{9}$, T. Kluge ${ }^{9}$, G. Knies ${ }^{9}$, A. Knutsson ${ }^{19}$, V. Korbel ${ }^{9}$, P. Kostka ${ }^{38}$, K. Krastev ${ }^{9}$,

J. Kretzschmar ${ }^{38}$, A. Kropivnitskaya ${ }^{24}$, K. Krüger ${ }^{13}$, M.P.J. Landon ${ }^{18}$, W. Lange ${ }^{38}$,

G. Laštovička-Medin ${ }^{30}$, P. Laycock ${ }^{17}$, A. Lebedev ${ }^{25}$, G. Leibenguth ${ }^{39}$, V. Lendermann ${ }^{13}$,

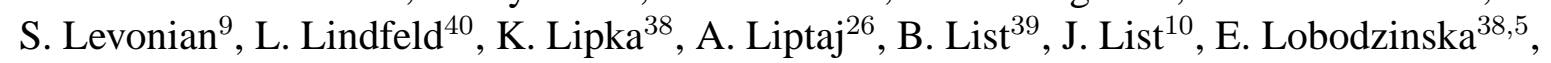

N. Loktionova ${ }^{25}$, R. Lopez-Fernandez ${ }^{23}$, V. Lubimov ${ }^{24}$, A.-I. Lucaci-Timoce ${ }^{9}$, H. Lueders ${ }^{10}$,

T. Lux $^{10}$, L. Lytkin ${ }^{11}$, A. Makankine ${ }^{7}$, N. Malden ${ }^{20}$, E. Malinovski $^{25}$, P. Marage ${ }^{3}$,

R. Marshall ${ }^{20}$, L. Marti ${ }^{9}$, M. Martisikova ${ }^{9}$, H.-U. Martyn ${ }^{1}$, S.J. Maxfi eld ${ }^{17}$, A. Mehta ${ }^{17}$,

K. Meier ${ }^{13}$, A.B. Meyer ${ }^{9}$, H. Meyer ${ }^{36}$, J. Meyer ${ }^{9}$, V. Michels ${ }^{9}$, S. Mikocki ${ }^{5}$,

I. Milcewicz-Mika $^{5}$, D. Milstead ${ }^{17}$, D. Mladenov ${ }^{34}$, A. Mohamed ${ }^{17}$, F. Moreau ${ }^{28}$,

A. Morozov ${ }^{7}$, J.V. Morris ${ }^{4}$, M.U. Mozer ${ }^{12}$, K. Müller ${ }^{40}$, P. Murín ${ }^{15,43}$, K. Nankov ${ }^{34}$,

B. Naroska ${ }^{10}$, Th. Naumann ${ }^{38}$, P.R. Newman ${ }^{2}$, C. Niebuhr ${ }^{9}$, A. Nikiforov ${ }^{26}$, G. Nowak ${ }^{5}$,

K. Nowak ${ }^{40}$, M. Nozicka ${ }^{32}$, R. Oganezov ${ }^{37}$, B. Olivier ${ }^{26}$, J.E. Olsson ${ }^{9}$, S. Osman ${ }^{19}$,

D. Ozerov ${ }^{24}$, V. Palichik ${ }^{7}$, I. Panagoulias ${ }^{9}$, T. Papadopoulou ${ }^{9}$, C. Pascaud ${ }^{27}$, G.D. Patel ${ }^{17}$,

H. Peng ${ }^{9}$, E. Perez ${ }^{8}$, D. Perez-Astudillo ${ }^{22}$, A. Perieanu ${ }^{9}$, A. Petrukhin ${ }^{24}$, D. Pitzl ${ }^{9}$,

R. Plačakyte ${ }^{26}$, B. Portheault ${ }^{27}$, B. Povh ${ }^{11}$, P. Prideaux ${ }^{17}$, A.J. Rahmat ${ }^{17}$, N. Raicevic ${ }^{30}$,

P. Reimer ${ }^{31}$, A. Rimmer ${ }^{17}$, C. Risler ${ }^{9}$, E. Rizvi ${ }^{18}$, P. Robmann ${ }^{40}$, B. Roland ${ }^{3}$, R. Roosen ${ }^{3}$,

A. Rostovtsev ${ }^{24}$, Z. Rurikova ${ }^{26}$, S. Rusakov ${ }^{25}$, F. Salvaire ${ }^{10}$, D.P.C. Sankey ${ }^{4}$, M. Sauter ${ }^{39}$,

E. Sauvan ${ }^{21}$, F.-P. Schilling ${ }^{9,44}$, S. Schmidt ${ }^{9}$, S. Schmitt ${ }^{9}$, C. Schmitz ${ }^{40}$, L. Schoeffel $^{8}$,

A. Schöning ${ }^{39}$, H.-C. Schultz-Coulon ${ }^{13}$, F. Sefkow ${ }^{9}$, R.N. Shaw-West ${ }^{2}$, I. Sheviakov ${ }^{25}$,

L.N. Shtarkov ${ }^{25}$, T. Sloan ${ }^{16}$, P. Smirnov ${ }^{25}$, Y. Soloviev ${ }^{25}$, D. South ${ }^{9}$, V. Spaskov ${ }^{7}$, A. Specka ${ }^{28}$,

M. Steder ${ }^{9}$, B. Stella ${ }^{33}$, J. Stiewe ${ }^{13}$, A. Stoilov ${ }^{34}$, U. Straumann ${ }^{40}$, D. Sunar ${ }^{3}$, V. Tchoulakov ${ }^{7}$,

G. Thompson ${ }^{18}$, P.D. Thompson ${ }^{2}$, T. Toll ${ }^{9}$, F. Tomasz ${ }^{15}$, D. Traynor ${ }^{18}$, T.N. Trinh ${ }^{21}$, P. Truöl $1^{40}$,

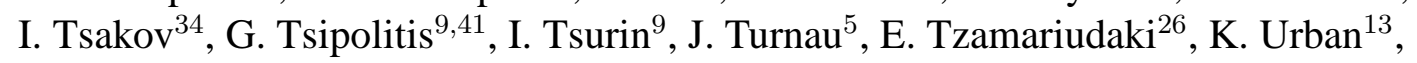

M. Urban ${ }^{40}$, A. Usik ${ }^{25}$, D. Utkin ${ }^{24}$, A. Valkárová ${ }^{32}$, C. Vallée ${ }^{21}$, P. Van Mechelen ${ }^{3}$, A. Vargas

Trevino $^{6}$, Y. Vazdik ${ }^{25}$, C. Veelken ${ }^{17}$, S. Vinokurova ${ }^{9}$, V. Volchinski ${ }^{37}$, K. Wacker ${ }^{6}$, G. Weber ${ }^{10}$, R. Weber ${ }^{39}$, D. Wegener ${ }^{6}$, C. Werner ${ }^{12}$, M. Wessels ${ }^{9}$, B. Wessling ${ }^{9}$, Ch. Wissing ${ }^{6}$, R. Wolf ${ }^{12}$, 
E. Wünsch ${ }^{9}$, S. Xella ${ }^{40}$, W. Yan ${ }^{9}$, V. Yeganov ${ }^{37}$, J. Žáček ${ }^{32}$, J. Zálešák ${ }^{31}, Z_{\text {Z. Zhang }}^{27}$, A. Zhelezov ${ }^{24}$, A. Zhokin ${ }^{24}$, Y.C. Zhu ${ }^{9}$, J. Zimmermann ${ }^{26}$, T. Zimmermann ${ }^{39}$, H. Zohrabyan ${ }^{37}$, and F. Zomer ${ }^{27}$

${ }^{1}$ I. Physikalisches Institut der RWTH, Aachen, Germany ${ }^{a}$

${ }^{2}$ School of Physics and Astronomy, University of Birmingham, Birmingham, UK ${ }^{b}$

${ }^{3}$ Inter-University Institute for High Energies ULB-VUB, Brussels; Universiteit Antwerpen,

Antwerpen; Belgium ${ }^{c}$

${ }^{4}$ Rutherford Appleton Laboratory, Chilton, Didcot, $U K^{b}$

${ }^{5}$ Institute for Nuclear Physics, Cracow, Poland ${ }^{2}$

${ }^{6}$ Institut für Physik, Universität Dortmund, Dortmund, Germany ${ }^{a}$

7 Joint Institute for Nuclear Research, Dubna, Russia

${ }^{8}$ CEA, DSM/DAPNIA, CE-Saclay, Gif-sur-Yvette, France

${ }^{9}$ DESY, Hamburg, Germany

${ }^{10}$ Institut für Experimentalphysik, Universität Hamburg, Hamburg, Germany ${ }^{a}$

${ }^{11}$ Max-Planck-Institut für Kernphysik, Heidelberg, Germany

${ }^{12}$ Physikalisches Institut, Universität Heidelberg, Heidelberg, Germany ${ }^{a}$

${ }^{13}$ Kirchhoff-Institut für Physik, Universität Heidelberg, Heidelberg, Germany ${ }^{a}$

${ }^{14}$ Institut für Experimentelle und Angewandte Physik, Universität Kiel, Kiel, Germany

${ }^{15}$ Institute of Experimental Physics, Slovak Academy of Sciences, Košice, Slovak Republic ${ }^{f}$

${ }^{16}$ Department of Physics, University of Lancaster, Lancaster, $U K^{b}$

${ }^{17}$ Department of Physics, University of Liverpool, Liverpool, $U K^{b}$

${ }^{18}$ Queen Mary and Westfield College, London, $U K^{b}$

${ }^{19}$ Physics Department, University of Lund, Lund, Sweden ${ }^{g}$

${ }^{20}$ Physics Department, University of Manchester, Manchester, $U K^{b}$

${ }^{21}$ CPPM, CNRS/IN2P3 - Univ. Mediterranee, Marseille - France

${ }^{22}$ Departamento de Fisica Aplicada, CINVESTAV, Mérida, Yucatán, México ${ }^{j}$

${ }^{23}$ Departamento de Fisica, CINVESTAV, México ${ }^{j}$

${ }^{24}$ Institute for Theoretical and Experimental Physics, Moscow, Russia ${ }^{k}$

${ }^{25}$ Lebedev Physical Institute, Moscow, Russia ${ }^{e}$

${ }^{26}$ Max-Planck-Institut für Physik, München, Germany

${ }^{27}$ LAL, Université de Paris-Sud 11, IN2P3-CNRS, Orsay, France

${ }^{28}$ LLR, Ecole Polytechnique, IN2P3-CNRS, Palaiseau, France

${ }^{29}$ LPNHE, Universités Paris VI and VII, IN2P3-CNRS, Paris, France

${ }^{30}$ Faculty of Science, University of Montenegro, Podgorica, Serbia and Montenegro ${ }^{e}$

${ }^{31}$ Institute of Physics, Academy of Sciences of the Czech Republic, Praha, Czech Republic ${ }^{h}$

${ }^{32}$ Faculty of Mathematics and Physics, Charles University, Praha, Czech Republic ${ }^{h}$

${ }^{33}$ Dipartimento di Fisica Università di Roma Tre and INFN Roma 3, Roma, Italy

${ }^{34}$ Institute for Nuclear Research and Nuclear Energy, Sofia, Bulgaria ${ }^{e}$

${ }^{35}$ Paul Scherrer Institut, Villigen, Switzerland

${ }^{36}$ Fachbereich C, Universität Wuppertal, Wuppertal, Germany

${ }^{37}$ Yerevan Physics Institute, Yerevan, Armenia

${ }^{38}$ DESY, Zeuthen, Germany

${ }^{39}$ Institut für Teilchenphysik, ETH, Zürich, Switzerland ${ }^{i}$

${ }^{40}$ Physik-Institut der Universität Zürich, Zürich, Switzerland ${ }^{i}$ 
${ }^{41}$ Also at Physics Department, National Technical University, Zografou Campus, GR-15773 Athens, Greece

${ }^{42}$ Also at Rechenzentrum, Universität Wuppertal, Wuppertal, Germany

${ }^{43}$ Also at University of P.J. Šafárik, Košice, Slovak Republic

${ }^{44}$ Also at CERN, Geneva, Switzerland

${ }^{45}$ Also at Max-Planck-Institut für Physik, München, Germany

${ }^{46}$ Also at Comenius University, Bratislava, Slovak Republic

${ }^{\dagger}$ Deceased

${ }^{a}$ Supported by the Bundesministerium für Bildung und Forschung, FRG, under contract numbers 05 H1 1GUA /1, 05 H1 1PAA /1, 05 H1 1PAB /9, 05 H1 1PEA /6, 05 H1 1VHA /7 and $05 \mathrm{HI} \mathrm{IVHB} / 5$

${ }^{b}$ Supported by the UK Particle Physics and Astronomy Research Council, and formerly by the UK Science and Engineering Research Council

${ }^{c}$ Supported by FNRS-FWO-Vlaanderen, IISN-IIKW and IWT and by Interuniversity Attraction Poles Programme, Belgian Science Policy

${ }^{d}$ Partially Supported by the Polish State Committee for Scientific Research, SPUB/DESY/P003/DZ 118/2003/2005

${ }^{e}$ Supported by the Deutsche Forschungsgemeinschaft

${ }^{f}$ Supported by VEGA SR grant no. 2/4067/24

${ }^{g}$ Supported by the Swedish Natural Science Research Council

${ }^{h}$ Supported by the Ministry of Education of the Czech Republic under the projects LC527 and INGO-1P05LA259

${ }^{i}$ Supported by the Swiss National Science Foundation

${ }^{j}$ Supported by CONACYT, México, grant 400073-F

${ }^{k}$ Partially Supported by Russian Foundation for Basic Research, grants 03-02-17291 and 04-02-16445 


\section{Introduction}

Diffractive processes such as $e p \rightarrow e X p$ have been studied extensively in deep-inelastic electron ${ }^{1}$-proton scattering (DIS) at the HERA collider [1-8], since understanding them in detail is fundamental to the development of quantum chromodynamics (QCD) at high parton densities. The photon virtuality $Q^{2}$ supplies a hard scale for the application of perturbative QCD, so that diffractive DIS events can be viewed as processes in which the photon probes a net colour singlet combination of exchanged partons. A hard scattering QCD collinear factorisation theorem [9] allows 'diffractive parton distribution functions' (DPDFs) to be defi ned, expressing proton parton probability distribution functions under the condition of a particular scattered proton four-momentum. The $x$ and $Q^{2}$ dependences of diffractive DIS can thus be treated with a similar theoretical description to that applied to inclusive DIS, for example through the application of the DGLAP parton evolution equations [10].

Within Regge phenomenology, diffractive cross sections are described by the exchange of a leading pomeron $(\mathbb{P})$ trajectory, as illustrated in fi gure 1. H1 diffractive DIS data [3] have been interpreted in a combined framework, which applies the QCD factorisation theorem to the $x$ and $Q^{2}$ dependences and uses a Regge inspired approach to express the dependence on the fraction $x_{\mathbb{P}}$ of the incident proton longitudinal momentum carried by the colour singlet exchange. The data at low $x_{\mathbb{P}}$ are well described in this framework and DPDFs and a pomeron trajectory intercept have been extracted. In order to describe the data at larger $x_{\mathbb{P}}$, it is necessary to include a sub-leading exchange trajectory $(\mathbb{R})$, with an intercept which is consistent [2] with the approximately degenerate trajectories associated with the $\rho, \omega, a_{2}$ and $f_{2}$ mesons.

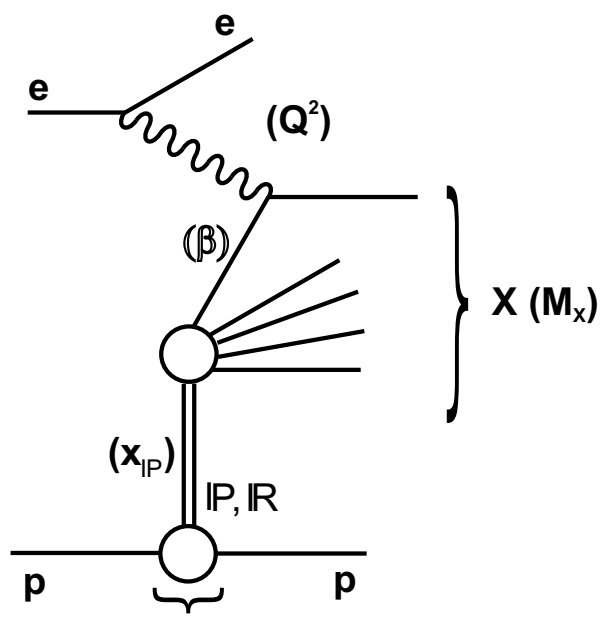

(t)

Figure 1: Schematic illustration of the diffractive DIS process $e p \rightarrow e X p$ and the kinematic variables used for its description in a model in which the pomeron $(\mathbb{P})$ and a sub-leading $(\mathbb{R})$ trajectory are exchanged.

In many previous analyses, including [3], diffractive DIS events are selected on the basis of the presence of a large rapidity gap (LRG) between the leading proton and the remainder

\footnotetext{
${ }^{1}$ For simplicity, the incident and scattered leptons are always referred to in the following as 'electrons', although the data studied here were obtained with both electron and positron beams.
} 
$X$ of the hadronic fi nal state. A complementary way to study diffractive processes is by direct measurement of the outgoing proton using the H1 Forward Proton Spectrometer (FPS) [7, 11] or its ZEUS counterpart [5]. Although the available statistics are smaller, the FPS method of studying diffraction has several advantages over the LRG method. In contrast to the LRG case, the squared four-momentum transfer at the proton vertex $t$ can be reconstructed. The FPS method also selects events in which the proton scatters elastically, whereas the LRG method does not distinguish the elastic case from dissociation to excited systems $Y$ with small masses $M_{Y}$. The FPS also allows measurements up to higher values of $x_{\mathbb{P}}$ than is possible with the LRG method, extending into regions where the sub-leading trajectory is the dominant exchange. Together, the FPS and LRG data thus provide a means of testing in detail the extent to which the variables $x_{\mathbb{P}}, t$ and $M_{Y}$ associated with the proton vertex can be factorised from the variables $\beta=x / x_{\mathbb{P}}$ and $Q^{2}$ describing the hard interaction.

In this paper, a measurement of the cross section for the diffractive DIS process $e p \rightarrow e X p$ using the FPS is reported. The $t$ dependence is presented in the form of a differential cross section $x_{\mathbb{P}} \mathrm{d}^{2} \sigma / \mathrm{d} t \mathrm{~d} x_{\mathbb{P}}$, from which the exponential slope of the $t$ distribution is measured and its dependence on other variables studied. Diffractive reduced cross sections, $\sigma_{r}^{D(4)}\left(\beta, Q^{2}, x_{\mathbb{P}}, t\right)$ at $|t|=0.25 \mathrm{GeV}^{2}$, and $\sigma_{r}^{D(3)}\left(\beta, Q^{2}, x_{\mathbb{P}}\right)$ integrated over $t$, are also measured. These observables are used to investigate the dependences on $\beta$ and $Q^{2}$, to extract the pomeron trajectory intercept from the $x_{\mathbb{P}}$ dependence and to quantify the sub-leading exchange contribution. The data are also compared directly with the LRG measurement [3] in order to test the compatibility between the results obtained with the two measurement techniques and to quantify the proton dissociation contribution in the LRG data.

\section{Experimental Technique}

The data used in this analysis correspond to an integrated luminosity of $28.4 \mathrm{pb}^{-1}$ and were collected with the H1 detector in the years 1999 and 2000. In these years the HERA collider was operated at electron and proton beam energies of $E_{e}=27.6 \mathrm{GeV}$ and $E_{p}=920 \mathrm{GeV}$, respectively, corresponding to an $e p$ centre of mass energy of $\sqrt{s}=319 \mathrm{GeV}$.

\subsection{H1 detector}

A detailed description of the $\mathrm{H} 1$ detector can be found elsewhere [12]. Here, the components most relevant for the present measurement are described briefly.

Scattered electrons with polar angles ${ }^{2}$ in the range $153^{\circ}<\theta_{e}^{\prime}<177^{\circ}$ are measured in a lead/scintillating-fi bre calorimeter, the SpaCal [13]. The energy resolution is $\sigma(E) / E \approx$ $7 \% / \sqrt{E[\mathrm{GeV}]} \oplus 1 \%$ and the energy scale uncertainty varies between $2.0 \%$ at a scattered electron energy of $E_{e}^{\prime}=11 \mathrm{GeV}$ and $0.5 \%$ at $E_{e}^{\prime}=27.6 \mathrm{GeV}$ [14]. A Backward Drift Chamber (BDC) in front of the SpaCal is used to measure the electron polar angle with a precision of

\footnotetext{
${ }^{2}$ In the right-handed coordinate system used, the origin is at the nominal interaction point, with the $+z$ axis and the polar angle $\theta=0$ in the direction of the outgoing proton beam (the 'forward' direction). The $+x$ axis points towards the centre of HERA. Transverse momenta are measured with respect to the beam axis.
} 
$0.5 \mathrm{mrad}$ and to suppress background where neutral particles fake the scattered electron signal. The SpaCal also has a hadronic section, with an energy scale known to a precision of $7 \%$.

The Central Tracking Detector (CTD), with a polar angle coverage of $20^{\circ}<\theta<160^{\circ}$, is used to reconstruct the interaction vertex and to measure the momentum of charged particles from the curvature of their trajectories in the $1.15 \mathrm{~T}$ fi eld provided by a superconducting solenoid. The fi nely segmented Liquid Argon (LAr) sampling calorimeter [15] surrounds the tracking system and covers the range in polar angle $4^{\circ}<\theta<154^{\circ}$. Its total depth varies with $\theta$ between 4.5 and 8 interaction lengths. The absolute hadronic energy scale is known with a precision of $4 \%$ for the measurements presented here. The hadronic fi nal state is reconstructed using an energy flow algorithm which combines charged particles measured in the CTD with information from the SpaCal and LAr calorimeters [16].

The luminosity is determined with a precision of $1.5 \%$ by detecting photons from the BetheHeitler process $e p \rightarrow e p \gamma$ in a crystal Čerenkov calorimeter, located at $z=-103 \mathrm{~m}$.

The energy and scattering angle of the leading proton are obtained from track measurements in the FPS $[7,11]$. Protons scattered through small angles are deflected by the proton beam-line magnets into a system of detectors placed within the proton beam pipe inside movable stations, known as Roman Pots. Each Roman Pot station contains four planes of fi ve scintillating fi bres, which together measure two orthogonal coordinates in the $(x, y)$ plane. The stations used in this analysis approach the beam horizontally from outside the proton ring and are positioned at $z=64 \mathrm{~m}$ and $z=80 \mathrm{~m}$. The detectors are sensitive to scattered protons which lose less than $10 \%$ of their energy in the $e p$ interaction and which are scattered through angles $\lesssim 1 \mathrm{mrad}$.

For each event, the leading proton energy and the proton scattering angles at the interaction point in the horizontal $(x-z)$ and vertical $(y-z)$ planes are obtained by applying transfer functions derived from the beam optics to the track parameters reconstructed in the FPS. The scattered proton energy is thus measured independently using the information in the horizontal and vertical planes. By comparison of these results, it is inferred that the energy resolution is around $6 \mathrm{GeV}$, independently of energy within the measured range, and that the absolute energy scale uncertainty is $0.5 \mathrm{GeV}$. The uncertainties in the reconstruction of the transverse momentum components $p_{x}$ and $p_{y}$ are quantifi ed using a sample of elastic $e p \rightarrow e \rho p$ photoproduction events with $\rho^{0} \rightarrow \pi^{+} \pi^{-}$decays. By comparing the FPS measurements with values reconstructed from the charged pions in the CTD, the resolution of the FPS is determined to be $\sim 40 \mathrm{MeV}$ for $p_{x}$ and $\sim 100 \mathrm{MeV}$ for $p_{y}$, dominated by the transverse momentum spread of the proton beam at the interaction point. The corresponding $t$-resolution varies over the measured range from $0.04 \mathrm{GeV}^{2}$ at $|t|=0.08 \mathrm{GeV}^{2}$ to $0.08 \mathrm{GeV}^{2}$ at $|t|=0.5 \mathrm{GeV}^{2}$. The uncertainties in the transverse momentum measurements are $10 \mathrm{MeV}$ for $p_{x}$ and $30 \mathrm{MeV}$ for $p_{y}$. The $t$-dependence measured in the FPS for the $\rho^{0}$ sample [17] is in good agreement with published H1 data [18]. For a leading proton which passes through both FPS stations, the average overall track reconstruction effi ciency is $20 \pm 2 \%$, corresponding to the product of the effi ciencies in the individual scintillating fi bre planes. The uncertainty on this effi ciency is evaluated by varying the details of the reconstruction procedure, for example the number of fi bres per plane which are required to register a track element. 


\subsection{Event selection and kinematic reconstruction}

The events used in this analysis are triggered on the basis of a coincidence between a track in the FPS, an electromagnetic cluster in the SpaCal calorimeter and a charged particle track providing an interaction vertex in the CTD. The trigger effi ciency varies with the kinematic variables studied and is around $85 \%$ on average.

Several selection criteria are applied to the data in order to suppress beam related backgrounds, background due to photoproduction processes and events in which the incoming electron loses signifi cant energy through QED radiation. The DIS selection criteria are summarised below.

- The reconstructed $z$ coordinate of the event vertex is required to lie within $35 \mathrm{~cm}$ $(\sim 3 \sigma)$ of the mean position. At least one track originating from the interaction vertex and reconstructed in the CTD is required to have a transverse momentum above $0.1 \mathrm{GeV}$.

- The variables characterising the scattered electron, $E_{e}^{\prime}$ and $\theta_{e}^{\prime}$, are determined from the SpaCal cluster, linked to a reconstructed charged particle track in the BDC, and the interaction vertex reconstructed in the CTD. The electron candidate is required to satisfy the criteria $155^{\circ}<\theta_{e}^{\prime}<176.5^{\circ}$ and $E_{e}^{\prime}>11 \mathrm{GeV}$.

- The quantity $E-p_{z}$, computed from the energies and longitudinal momenta of all reconstructed particles including the electron, is required to lie between $35 \mathrm{GeV}$ and $70 \mathrm{GeV}$. Neglecting detector effects and QED radiation, this quantity is expected to be twice the electron beam energy for neutral current DIS events.

The following requirements are applied to the leading proton measured in the FPS.

- The measurement is restricted to the region where the FPS acceptance is high by requiring that the transverse momenta in the horizontal and vertical projections lie in the ranges $-0.38<p_{x}<-0.24 \mathrm{GeV}$ and $\left|p_{y}\right|<0.7 \mathrm{GeV}$, respectively, and that the fractional energy of the leading proton, $E_{p}^{\prime} / E_{p}$, be greater than 0.9 .

- To suppress cases where a DIS event reconstructed in the central detector coincides with background in the FPS, for example due to an off-momentum beam proton (beam halo), the quantity $E+p_{z}$, summed over all reconstructed particles including the leading proton, is required to be below $1900 \mathrm{GeV}$. Neglecting detector effects, this quantity is expected to be twice the proton beam energy for neutral current DIS events.

The inclusive DIS kinematic variables, $Q^{2}, x$ and the inelasticity $y$, are reconstructed using the techniques introduced in [2]. In order to optimise the resolution throughout the measured $y$ range, information is exploited from both the scattered electron and the hadronic fi nal state according to

$$
y=y_{e}^{2}+y_{d}\left(1-y_{d}\right) \quad ; \quad Q^{2}=\frac{4 E_{e}^{2}(1-y)}{\tan ^{2}\left(\theta_{e}^{\prime} / 2\right)} \quad ; \quad x=\frac{Q^{2}}{s y} .
$$


Here, $y_{e}$ and $y_{d}$ denote the values of $y$ obtained from the scattered electron only ('electron method') and from the angles of the electron and the hadronic fi nal state ('double angle method'), respectively [19]. The analysis is restricted to the region $2<Q^{2}<50 \mathrm{GeV}^{2}$ and $0.02<y<$ 0.6 .

With $q, P$ and $P^{\prime}$ denoting the four-vectors of the exchanged virtual photon and the incoming and outgoing protons, respectively, further variables specifi c to diffractive DIS are defi ned as

$$
x_{\mathbb{P}}=\frac{q \cdot\left(P-P^{\prime}\right)}{q \cdot P} ; \beta=\frac{Q^{2}}{2 q \cdot\left(P-P^{\prime}\right)},
$$

such that $\beta$ can be interpreted as the fraction of the colourless exchange longitudinal momentum which is carried by the struck quark. Two different methods are used to reconstruct these variables. In the 'leading proton' method, $x_{\mathbb{P}}$ is reconstructed directly from the energy of the leading proton, such that

$$
x_{\mathbb{P}}=1-E_{p}^{\prime} / E_{p} \quad ; \quad \beta=\frac{x}{x_{\mathbb{P}}} .
$$

In the ' $X$-mass' method, the mass of the system $X$ is fi rst obtained from the hadrons reconstructed in the central detector using

$$
M_{X}^{2}=\left(E^{2}-p_{x}^{2}-p_{y}^{2}-p_{z}^{2}\right)_{\mathrm{had}} \cdot \frac{y}{y_{h}},
$$

where the subscript 'had' represents a sum over all hadronic fi nal state particles excluding the leading proton and $y_{h}$ is the value of $y$ reconstructed using only the hadronic fi nal state [20]. Including the factor $y / y_{h}$ leads to cancellations of many measurement inaccuracies. The diffractive variables are then reconstructed using

$$
\beta=\frac{Q^{2}}{Q^{2}+M_{X}^{2}} \quad ; \quad x_{\mathbb{P}}=\frac{x}{\beta} .
$$

The results obtained with the leading proton and $X$-mass methods agree well in the low $x_{\mathbb{P}}$ range where both are applicable. The $X$-mass method is used for $x_{\mathbb{P}}<0.006$ and the leading proton method is used for $x_{\mathbb{P}}>0.006$, the choice being made on the basis of which method provides the better resolution.

The squared four-momentum transfer $t=\left(P-P^{\prime}\right)^{2}$ is reconstructed using the transverse momentum $p_{t}$ of the leading proton measured with the FPS and the best value of $x_{\mathbb{P}}$ as described above, such that

$$
t=t_{\min }-\frac{p_{t}^{2}}{1-x_{\mathbb{P}}} ; t_{\min }=-\frac{x_{\mathbb{P}}^{2} m_{p}^{2}}{1-x_{\mathbb{P}}}
$$

where $\left|t_{\text {min }}\right|$ is the minimum kinematically accessible value of $|t|$ and $m_{p}$ is the proton mass. In the analysis, the reconstructed $|t|$ is required to lie in the range $0.08<|t|<0.5 \mathrm{GeV}^{2}$. The fi nal data sample contains about 3300 events. 


\section{Monte Carlo simulation and corrections to the data}

Monte Carlo simulations are used to correct the data for the effects of detector acceptances and ineffi ciencies, migrations between measurement intervals due to fi nite resolutions and QED radiation. The reaction $e p \rightarrow e X p$ is simulated using an implementation of the 'saturation' model [21] within the RAPGAP generator [22]. Following hadronisation using the Lund string model [23] as implemented in JETSET [24], the response of the H1 detector is simulated in detail and the events are passed through the same analysis chain as is used for the data. Weights are applied to the generated events so that the important kinematic variable distributions are well described throughout the region of the measurement.

The background from photoproduction processes, where the electron is scattered into the backward beampipe and a particle from the hadronic fi nal state fakes the electron signature in the SpaCal, is estimated using the PHOJET Monte Carlo model [25]. This background is negligible except at the highest $y$ values and is $6 \%$ at most. The proton dissociation background, where the leading proton originates from the decay of a higher mass state, is estimated using an implementation in RAPGAP of the dissociation model originally developed for the DIFFVM Monte Carlo generator [26]. This background is negligible except at the highest $x_{\mathbb{P}}$ values, where it reaches $2.7 \%$. Background also arises from random coincidences of DIS events causing activity in the central detector with beam-halo protons giving a signal in the FPS. This contribution is estimated statistically by combining DIS events (without the requirement of a track in the FPS) with beam-halo protons from randomly triggered events. Subtractions of up to $7 \%$ are made as a function of the total reconstructed $E+p_{z}$ of the event.

Cross sections are obtained at the Born level, using RAPGAP interfaced to the program HERACLES [27] to correct for QED radiative effects. The data are presented at fi xed $Q^{2}$, $\beta, x_{\mathbb{P}}$ and $t$ values, with corrections applied for the influence of the fi nite bin sizes using a parameterisation of the '2006 DPDF Fit A' to the H1 LRG data [3] for the $Q^{2}, \beta$ and $x_{\mathbb{P}}$ dependences and the $t$ dependences measured in this analysis at each $x_{\mathbb{P}}$ value (see section 5.1).

\section{Systematic Uncertainties on the Measured Cross Sections}

Systematic uncertainties are considered from the following sources.

- The uncertainties in the leading proton energy, its transverse momentum in the horizontal projection and that in the vertical projection are $0.5 \mathrm{GeV}, 10 \mathrm{MeV}$ and $30 \mathrm{MeV}$, respectively (see section 2.1). The corresponding average uncertainties on the $\sigma_{r}^{D(3)}$ and $\sigma_{r}^{D(4)}$ measurements are $5.7 \%, 6.0 \%$ and $3.3 \%$.

- The energy scale uncertainty of the SpaCal implies an error of between $0.5 \%$ and $2.0 \%$ (depending on the energy) on the $E_{e}^{\prime}$ measurement, which leads to an average systematic error of $3.0 \%$ on the $\sigma_{r}^{D}$ data points. Possible biases in the $\theta_{e}^{\prime}$ measurement at the level of $\pm 0.5 \mathrm{mrad}$ lead to an average systematic error of $2.4 \%$. 
- The systematic uncertainties arising from the hadronic fi nal state reconstruction are determined by varying the hadronic energy scales of the LAr and SpaCal calorimeters by $4 \%$ and $7 \%$, respectively, and the energy fraction carried by tracks by $3 \%$. Each of these sources leads to an uncertainty in the $\sigma_{r}^{D}$ measurements of typically $1.5 \%$.

- The model dependence of the acceptance and migration corrections is estimated by varying the shapes of the distributions in the kinematic variables $x_{\mathbb{P}}, \beta$ and $t$ in the RAPGAP simulation within the limits imposed by the present data. The $x_{\mathbb{P}}$ distribution is reweighted by $\left(1 / x_{\mathbb{P}}\right)^{ \pm 0.1}$, which leads to an average uncertainty of $2.0 \%$ in $\sigma_{r}^{D}$. The $\beta$ distribution is reweighted by $\beta^{ \pm 0.1}$ and $(1-\beta)^{ \pm 0.1}$, leading to typical uncertainties of $3.2 \%$. Reweighting the $t$ distribution by $e^{ \pm t}$ results in uncertainties of $2.5 \%$ on average.

- The uncertainties related to the subtraction of backgrounds (see section 3) are at most $2.7 \%$ for proton dissociation, $3.0 \%$ for photoproduction and $3.5 \%$ for the proton beamhalo contribution.

- A $2.6 \%$ uncertainty is attributed to the trigger effi ciencies (section 2.2), evaluated using independent triggers.

- The uncertainty in the FPS track reconstruction effi ciency results in an overall normalisation uncertainty of $10 \%$ (see section 2.1). A further normalisation uncertainty of $1.5 \%$ arises from the luminosity measurement.

- The extrapolation from the measured FPS range of $0.08<|t|<0.5 \mathrm{GeV}^{2}$ to the region $\left|t_{\text {min }}\right|<|t|<1 \mathrm{GeV}^{2}$ covered by the LRG data [3] results in an additional systematic error of up to $5 \%$ for the $\sigma_{r}^{D(3)}$ data (see section 5.3).

The systematic errors shown in the fi gures and tables are calculated as the quadratic sum of all contributions which vary from point to point, corresponding to average uncertainties of $12 \%$ for the $\sigma_{r}^{D(4)}$ data and $13 \%$ for $\sigma_{r}^{D(3)}$. The quoted errors do not include the overall normalisation uncertainty.

\section{Results and Discussion}

\subsection{Cross section dependence on $t$}

The differential cross section $\mathrm{d}^{2} \sigma / \mathrm{d} x_{\mathbb{P}} \mathrm{d} t$ provides a measurement of the $t$ dependence of diffractive DIS. This cross section is shown in fi gure $2 \mathrm{a}$, multiplied by $x_{\mathbb{P}}$ for convenience, for three values of $t$ and six values of $x_{\mathbb{P}}$ in the range $x_{\mathbb{P}}<0.1$ and $0.08<|t|<0.5 \mathrm{GeV}^{2}$, integrated over $2<Q^{2}<50 \mathrm{GeV}^{2}$ and $0.02<y<0.6$. For each $x_{\mathbb{P}}$ value, fi ts to the form $x_{\mathbb{P}} \mathrm{d}^{2} \sigma / \mathrm{d} x_{\mathbb{P}} \mathrm{d} t \propto e^{B t}$ are shown in fi gure $2 \mathrm{a}$. The extracted values of the slope parameter $B$ are plotted as a function of $x_{\mathbb{P}}$ in fi gure $2 \mathrm{~b}$ and are listed in table 3 . The H1 results for $B$ are consistent with ZEUS measurements [5], though the H1 data are somewhat lower than the ZEUS data for $x_{\mathbb{P}} \lesssim 0.02$. 
At low $x_{\mathbb{P}}$, the data are compatible with a constant slope parameter, $B \simeq 6 \mathrm{GeV}^{2}$. In a Regge approach with a single linear exchanged trajectory, $\alpha_{\mathbb{P}}(t)=\alpha_{\mathbb{P}}(0)+\alpha_{\mathbb{P}}^{\prime} t$, the slope parameter is expected to decrease logarithmically with increasing $x_{\mathbb{P}}$ according to

$$
B=B_{\mathbb{P}}-2 \alpha_{\mathbb{P}}^{\prime} \ln x_{\mathbb{P}},
$$

an effect which is often referred to as 'shrinkage' of the diffractive peak. The degree of shrinkage depends on the slope of the pomeron trajectory, which is $\alpha_{\mathbb{I P}}^{\prime} \simeq 0.25 \mathrm{GeV}^{-2}$ for soft hadronhadron scattering at high energies [28]. In contrast, vector meson measurements at HERA have resulted in smaller values of $\alpha_{\mathbb{P}}^{\prime}$, whether a hard scale is present [29,30] or not [31]. Fits of the form of equation 7 are performed to the FPS data shown in fi gure $2 b$ in the region where pomeron exchange is expected to dominate, namely to the three data points with $0.0009 \leq$ $x_{\mathbb{P}} \leq 0.0094$, for which the sub-leading exchange contribution is estimated to be at most $7 \%$ (see the fit results in section 5.2). A two parameter fit to the data in this range yields $R_{\mathbb{P}}=$ $6.0 \pm 1.6$ (stat.) ${ }_{-1.0}^{+2.4}$ (syst.) $\mathrm{GeV}^{-2}$ and $\alpha_{\mathbb{P}}^{\prime}=0.02 \pm 0.14$ (stat.) ${ }_{-0.09}^{+0.21}$ (syst.) $\mathrm{GeV}^{-2}$. Extending the fi t range to the interval $0.0009 \leq x_{\mathbb{P}} \leq 0.021$, for which the contribution of the sub-leading exchange is at most $20 \%$ (section 5.2), results in $B_{\mathbb{P}}=4.9 \pm 1.2$ (stat.) ${ }_{-0.7}^{+1.6}$ (syst.) $\mathrm{GeV}^{-2}$ and $\alpha_{\mathbb{P}}^{\prime}=0.10 \pm 0.10$ (stat.) ${ }_{-0.07}^{+0.16}$ (syst.) $\mathrm{GeV}^{-2}$. The data thus favour a small value of $\alpha^{\prime}$, as expected in perturbative models of the pomeron [32]. However, the result $\alpha_{\mathbb{P}}^{\prime} \simeq 0.25$ from soft interactions cannot be excluded. The results of these fi ts are summarised in table 1.

\begin{tabular}{|c|c|c|}
\hline Range of Fit & $\alpha_{\mathbb{P}}^{\prime}\left(\mathrm{GeV}^{-2}\right)$ & $B_{\mathbb{P}}\left(\mathrm{GeV}^{-2}\right)$ \\
\hline $0.0009 \leq x_{\mathbb{P}} \leq 0.0094$ & $0.02 \pm 0.014_{-0.09}^{+0.21}$ & $6.0 \pm 1.6_{-1.0}^{+2.4}$ \\
$0.0009 \leq x_{\mathbb{P}} \leq 0.021$ & $0.10 \pm 0.010_{-0.07}^{+0.16}$ & $4.9 \pm 1.2_{-0.7}^{+1.6}$ \\
\hline
\end{tabular}

Table 1: The results of fi ts to the slope parameter data in two different ranges at low $x_{\mathbb{P}}$ in order to extract $\alpha_{\mathbb{P}}^{\prime}$ and $B_{\mathbb{P}}$, together with their statistical (fi rst error) and systematic (second error) uncertainties.

A decrease of the slope $B$ is observed towards the region of larger $x_{\mathbb{P}} \gtrsim 0.03$, where the contribution from the sub-leading exchange is expected to be signifi cant $(60 \%$ in the highest bin at $x_{\mathbb{P}}=0.076$ ). This reduction of the slope parameter indicates that the size of the interaction region reduces as $x_{\mathbb{P}}$ increases, reaching values of around $4 \mathrm{GeV}^{-2}$, characteristic of the spatial extent of the proton charge distribution.

The $t$ dependence of the cross section is also presented in fi gure 3 and table 4 in different regions of $Q^{2}$ and $\beta$ for two $x_{\mathbb{P}}$ intervals. No signifi cant $Q^{2}$ or $\beta$ dependence of the slope parameter $B$ is observed for $x_{\mathbb{P}}<0.03$. Within the uncertainties, the $t$ dependence of the cross section in the pomeron dominated low $x_{\mathbb{P}}$ region can therefore be factorised from the $Q^{2}$ and $\beta$ dependences. Since there is also no strong evidence for any $\beta$ or $Q^{2}$ dependence of $B$ for $x_{\mathbb{P}}>0.03$, the data are consistent with a similar factorisation for the sub-leading exchange contribution.

\subsection{Cross section dependence on $x_{\mathbb{P}}$ and extraction of $\alpha_{\mathbb{P}}(0)$}

The $x_{\mathbb{P}}, \beta$ and $Q^{2}$ dependences of diffractive DIS are studied in terms of the diffractive reduced cross sections $\sigma_{r}^{D(4)}$ and $\sigma_{r}^{D(3)}$. The former observable is related to the measured differential 
cross section by [3]

$$
\frac{\mathrm{d}^{4} \sigma^{e p \rightarrow e X p}}{\mathrm{~d} \beta d Q^{2} \mathrm{~d} x_{\mathbb{P}} \mathrm{d} t}=\frac{4 \pi \alpha^{2}}{\beta Q^{4}} \cdot\left(1-y+\frac{y^{2}}{2}\right) \cdot \sigma_{r}^{D(4)}\left(\beta, Q^{2}, x_{\mathbb{P}}, t\right) .
$$

The reduced cross section is equal to the diffractive structure function $F_{2}^{D(4)}\left(\beta, Q^{2}, x_{\mathbb{P}}, t\right)$ to good approximation in the relatively low $y$ region covered by the current analysis, where the contribution from the longitudinal structure function $F_{L}^{D(4)}\left(\beta, Q^{2}, x_{\mathbb{P}}, t\right)$ is small. Results for $\sigma_{r}^{D(4)}$ are obtained at a fi xed value of $|t|=0.25 \mathrm{GeV}^{2}$, interpolating from the measured range $0.08<|t|<0.5 \mathrm{GeV}^{2}$ using the measured $t$ dependence at each $x_{\mathbb{P}}$ value (fi gure 2 ). Presenting the measurement at $|t|=0.25 \mathrm{GeV}^{2}$ ensures that the systematic uncertainties associated with this interpolation are small.

Figure 4 shows $x_{\mathbb{P}} \sigma_{r}^{D(4)}$ for $|t|=0.25 \mathrm{GeV}^{2}$ as a function of $x_{\mathbb{P}}$ for different $Q^{2}$ and $\beta$ values (see also tables 5- 8). At medium and large $\beta$ values, $x_{\mathbb{P}} \sigma_{r}^{D(4)}$ falls or is flat as a function of $x_{\mathbb{P}}$. Qualitatively this behaviour is consistent with a dominant pomeron contribution with an intercept $\alpha_{\mathbb{P}}(0) \gtrsim 1$. However, $x_{\mathbb{P}} \sigma_{r}^{D(4)}$ rises with $x_{\mathbb{P}}$ at the highest $x_{\mathbb{P}}$ for low $\beta$ values, which can be explained by a contribution from a sub-leading exchange with an intercept $\alpha_{\mathbb{R}}(0)<1$.

To describe the $x_{\mathbb{P}}$ dependence quantitatively, a fit is performed to the structure function $F_{2}^{D(4)}$, obtained by correcting $\sigma_{r}^{D(4)}$ for the small $F_{L}^{D(4)}$ contribution using the results of the '2006 DPDF fi t A' in [3]. A parameterisation of the form

$$
F_{2}^{D(4)}=f_{\mathbb{P}}\left(x_{\mathbb{P}}, t\right) F_{\mathbb{P}}\left(\beta, Q^{2}\right)+n_{\mathbb{R}} \cdot f_{\mathbb{R}}\left(x_{\mathbb{P}}, t\right) F_{\mathbb{R}}\left(\beta, Q^{2}\right)
$$

is used. This parameterisation assumes a separate 'proton vertex' factorisation of the $x_{\mathbb{P}}$ and $t$ dependences from those on $\beta$ and $Q^{2}$ for both the pomeron and a sub-leading exchange. The factors $f_{\mathbb{P}}$ and $f_{\mathbb{R}}$ correspond to flux factors for the exchanges and are taken from the Reggemotivated functions,

$$
f_{\mathbb{P}}\left(x_{\mathbb{P}}, t\right)=A_{\mathbb{P}} \cdot \frac{e^{B_{\mathbb{P}} t}}{x_{\mathbb{P}}^{2 \alpha_{\mathbb{P}}(t)-1}} \quad ; \quad f_{\mathbb{R}}\left(x_{\mathbb{P}}, t\right)=A_{\mathbb{R}} \cdot \frac{e^{B_{\mathbb{R}} t}}{x_{\mathbb{P}}^{2 \alpha_{\mathbb{R}}(t)-1}},
$$

assuming that the sub-leading exchange has a linear trajectory $\alpha_{\mathbb{R}}(t)=\alpha_{\mathbb{R}}(0)+\alpha_{\mathbb{R}}^{\prime} t$ as for the pomeron. The values of $A_{\mathbb{P}}$ and $A_{\mathbb{R}}$ are chosen such that $x_{\mathbb{P}} \cdot \int_{-1}^{t_{\min }} f_{\mathbb{P}, \mathbb{R}}\left(x_{\mathbb{P}}, t\right) \mathrm{d} t=1$ at $x_{\mathbb{P}}=0.003$, following the convention of [2]. The free parameters of the fit are the pomeron intercept $\alpha_{\mathbb{P}}(0)$, normalisation coeffi cients $F_{\mathbb{P}}\left(\beta, Q^{2}\right)$ for the pomeron contribution at each of the nineteen $\left(\beta, Q^{2}\right)$ values considered, and a single parameter $n_{\mathbb{R}}$ describing the normalisation of the sub-leading exchange contribution.

A summary of the values assumed for the parameters which are fi xed in the fi ts is given in table 2. The intercept $\alpha_{\mathbb{R}}(0)$ of the sub-leading exchange is obtained from [2]. As in [2,3], the normalisation coeffi cients $F_{\mathbb{R}}\left(\beta, Q^{2}\right)$ for the sub-leading exchange in each $\beta$ and $Q^{2}$ bin are taken from a parameterisation of the pion structure function [33]. The remaining fi xed parameters describing the fluxes are taken from the present analysis. Averages of the two fi ts to the $B\left(x_{\mathbb{P}}\right)$ data at low $x_{\mathbb{P}}$ described in section 5.1 (table 1) are used to fix the pomeron parameters, $B_{\mathbb{P}}=5.5 \mathrm{GeV}^{-2}$ and $\alpha_{\mathbb{P}}^{\prime}=0.06 \mathrm{GeV}^{-2}$. The behaviour of $B\left(x_{\mathbb{P}}\right)$ at large $x_{\mathbb{P}}$ is sensitive to the parameters $\alpha_{\mathbb{R}}^{\prime}$ and $B_{\mathbb{R}}$. Although the constraints are not strong, the data 
are incompatible with the pair of values, $\alpha_{\mathbb{R}}^{\prime}=0.9 \mathrm{GeV}^{-2}[34]$ and $B_{\mathbb{R}}=2.0 \mathrm{GeV}^{-2}$ [35], obtained from soft hadronic scattering data and applied previously in similar fi ts to $F_{2}^{D}$ data [2]. A good description of the slope parameter results over the full $x_{\mathbb{P}}$ range is obtained with $B_{\mathbb{R}}=$ $1.6 \mathrm{GeV}^{-2}$ and $\alpha_{\mathbb{R}}^{\prime}=0.3 \mathrm{GeV}^{-2}$. A description based on this parameterisation is shown in fi gure $2 b$.

\begin{tabular}{|l|c|}
\hline Parameter & Value \\
\hline$\alpha_{\mathbb{P}}^{\prime}$ & $0.06_{-0.06}^{+0.19} \mathrm{GeV}^{-2}$ \\
$B_{\mathbb{P}}$ & $5.5_{+0.7}^{-2.0} \mathrm{GeV}^{-2}$ \\
$\alpha_{\mathbb{R}}(0)$ & $0.50 \pm 0.10$ \\
$\alpha_{\mathbb{R}}^{\prime}$ & $0.3_{-0.3}^{+0.6} \mathrm{GeV}^{-2}$ \\
$B_{\mathbb{R}}$ & $1.6_{+0.4}^{-1.6} \mathrm{GeV}^{-2}$ \\
\hline
\end{tabular}

Table 2: The values of the fi xed parameters and their uncertainties, as used in the extraction of $\alpha_{\mathbb{P}}(0)$. Since $\alpha_{\mathbb{P}}^{\prime}$ and $B_{\mathbb{P}}$ are strongly anti-correlated when extracted from the data shown in fi gure $2 \mathrm{~b}$, they are varied simultaneously to obtain the errors on the fi t results, as are $\phi_{R}$ and $B_{\mathbb{R}}$.

The experimental systematic uncertainties on the free parameters are evaluated by repeating the fi $\mathrm{t}$ after shifting the data points according to each individual uncertainty source described in section 4. A model dependence uncertainty is determined by varying the fi xed parameters as described in table 2. The $\alpha_{\mathbb{P}, \mathbb{R}}^{\prime}$ and $B_{\mathbb{P}, \mathbb{R}}$ parameters are varied in the ranges given in the table, within which an acceptable description of the data is maintained, whilst requiring that $\alpha_{I P}^{\prime}$ and $\alpha_{I R}^{\prime}$ lie between 0 and the values describing soft hadronic scattering $\left(0.25 \mathrm{GeV}^{-2}\right.$ and $0.9 \mathrm{GeV}^{-2}$, respectively). The influence of neglecting the $F_{L}^{D(4)}$ contribution is also included in the model dependence uncertainty.

As shown in fi gure 4, the fi t provides a good description of the xip dependence of the data ( $\chi^{2}=44$ with statistical uncertainties for 51 degrees of freedom). Within uncertainties, the $x_{\mathbb{P}}$ dependence can therefore be factorised from the $\beta$ and $Q^{2}$ dependences for each of the pomeron and the sub-leading contributions.

The fi $t$ yields a pomeron intercept of

$$
\alpha_{\mathbb{P}}(0)=1.114 \pm 0.018 \text { (stat.) } \pm 0.012 \text { (syst.) }{ }_{-0.020}^{+0.040} \text { (model) },
$$

the dominant uncertainty arising from the variations of $\alpha_{\mathbb{P}}^{\prime}$ and $B_{\mathbb{P}}$. This result for $\alpha_{\mathbb{P}}(0)$ is compatible with that obtained from H1 data measured using the LRG method [3] and with ZEUS measurements $[5,6]$. It is only slightly higher than the pomeron intercept describing soft hadronic scattering, $\alpha_{\mathbb{P}}(0) \simeq 1.08$ [28]. However, if $\alpha_{\mathbb{P}}^{\prime}$ is set to the soft pomeron value of $0.25 \mathrm{GeV}^{-2}, \alpha_{\mathbb{P}}(0)$ increases to around 1.15 .

The result for the sub-leading exchange normalisation parameter is

$$
n_{\mathbb{R}}=\left[1.0 \pm 0.2 \text { (stat.) } \pm 0.1 \text { (syst.) }{ }_{-0.7}^{+1.2}(\text { model })\right] \times 10^{-3},
$$

the largest uncertainty arising from the variation of $\alpha_{\mathbb{R}}(0)$. The sub-leading exchange is important at low $\beta$ and high $x_{\mathbb{P}}$, contributing typically $60 \%$ of the cross section at the highest $x_{\mathbb{P}}=0.08$. 


\subsection{Cross section dependence on $Q^{2}$ and $\beta$}

The reduced cross section $\sigma_{r}^{D(3)}\left(\beta, Q^{2}, x_{\mathbb{P}}\right)$ is defi ned as the integral of $\sigma_{r}^{D(4)}\left(\beta, Q^{2}, x_{\mathbb{P}}, t\right)$ over the range $\left|t_{\text {min }}\right|<|t|<1 \mathrm{GeV}^{2}$, which is the region covered by H1 using the LRG method [3]. It is obtained here by extrapolating the FPS data from the measured range $0.08<$ $|t|<0.5 \mathrm{GeV}^{2}$ using the $t$ dependence at each $x_{\mathbb{P}}$ value (section 5.1 and table 3 ). The extrapolation factor depends only weakly on $x_{\mathbb{P}}$ and is 1.7 on average, with an uncertainty of up to $5 \%$. The measurement of $x_{\mathbb{P}} \sigma_{r}^{D(3)}$ is presented in fi gures 5-7 and tables 5- 8. The data are compared with predictions derived from the '2006 DPDF Fit A' to the LRG data presented in [3], with modifi cations as described in section 6.1.

The $Q^{2}$ dependence of $\sigma_{r}^{D(3)}$ at fi xed $x_{\mathbb{P}}$ and $\beta$ (fi gure 5) is characterised by positive scaling violations $\left(\partial \sigma_{r}^{D(3)} / \partial \ln Q^{2}>0\right)$ throughout the kinematic range, except possibly at the highest $\beta=0.7$. This observation is consistent with that from $\mathrm{H} 1$ measurements using the LRG method [2,3] and implies a large gluonic component to the DPDFs. As can be seen from the model comparison, the positive scaling violations may be attributed to the pomeron contribution even at the highest $x_{\mathbb{P}}$ values, where the sub-leading exchange is dominant.

The dependence of $\sigma_{r}^{D(3)}$ on $\beta$ is weak over most of the kinematic range (fi gure 6). Since the $\beta$ dependence is determined in the quark-parton model by the diffractive quark densities, this implies that the quark densities do not decrease at the highest values of $\beta$ studied. Indeed, $\sigma_{r}^{D(3)}$ clearly rises as $\beta \rightarrow 1$ at low $Q^{2}$ and $x_{\mathbb{P}}$. Within the framework of DPDFs, this can be explained in terms of diffractive quark densities peaking at high fractional momenta at low $Q^{2}[2,3]$. The $\beta$ dependence of diffractive DIS has also been interpreted in terms of the elastic scattering from the proton of colour dipoles produced by partonic fluctuations of the virtual photon [21,36,37]. In such models, the cross section at low and intermediate $\beta$ values is dominated by $q \bar{q} g$ and $q \bar{q}$ fluctuations of transversely polarised photons, respectively. The rise of $\sigma_{r}^{D(3)}$ as $\beta \rightarrow 1$ at low $Q^{2}$ has been interpreted in terms of $q \bar{q}$ fluctuations of longitudinally polarised photons [38], which are suppressed as $Q^{2}$ increases.

\section{Comparison with Other Measurements}

\subsection{Comparison with $\mathrm{H1}$ large rapidity gap data.}

The FPS $\sigma_{r}^{D(3)}$ data can be compared with H1 measurements obtained using the LRG technique [3], after taking into account the slightly different cross section defi nitions in the two cases. Firstly, the cross section $e p \rightarrow e X Y$ measured with the LRG data is defi ned to include proton dissociation to any system $Y$ with a mass in the range $M_{Y}<1.6 \mathrm{GeV}$, whereas $Y$ is defi ned to be a proton in the cross section measured with the FPS. Secondly, if there are signifi cant isospin- 1 contributions to the sub-leading trajectory, charge-exchange reactions producing leading neutrons are expected in the LRG measurement, which are not present in the proton-tagged FPS data.

A point-by point comparison between the $\sigma_{r}^{D(3)}$ data obtained with the LRG and FPS methods can be found in [3]. Here, the level of agreement is scrutinised in more detail in the range 
$x_{\mathbb{P}} \lesssim 0.05$, to which the LRG method is applicable. To make the comparison with a minimum of systematic uncertainty and to test for differences between the kinematic dependences of the two cross sections, the LRG measurement is repeated with an identical $Q^{2}, \beta$ and $x_{\mathbb{P}}$ binning to that used for the FPS data. The ratio of the two measurements is formed for each $\left(Q^{2}, \beta\right.$, $x_{\mathbb{P}}$ ) point and the dependences of this ratio on each kinematic variable individually is studied by taking statistically weighted averages over the other two variables. Since the two data sets are statistically independent and the dominant sources of systematic error are very different, correlations between the uncertainties on the FPS and LRG data are neglected.

The ratio of the LRG to the FPS cross section is plotted in fi gure 8 as a function of $Q^{2}, \beta$ and $x_{\mathbb{P}}$. The combined normalisation errors of $12.7 \%$ are not shown. Within the remaining uncertainties of typically $10 \%$ per data point, there is no signifi cant dependence on $\beta, Q^{2}$ or $x_{I P}$. The ratio of overall normalisations, LRG / FPS, is

$$
\frac{\sigma\left(M_{Y}<1.6 \mathrm{GeV}\right)}{\sigma(Y=p)}=1.23 \pm 0.03 \text { (stat.) } \pm 0.16 \text { (syst.) }
$$

the dominant uncertainties arising from the normalisations of the FPS and LRG data. This result is consistent with the prediction of $1.15_{-0.08}^{+0.15}$ from the DIFFVM generator, where the total proton-elastic and proton dissociation cross sections are taken to be equal by default and their ratio is varied in the range $1: 2$ to $2: 1$ for the uncertainties $[3,26]$.

Since the FPS measurement extends to larger $x_{\mathbb{I P}}$ values than the LRG measurement, the FPS data provide complementary constraints on the sub-leading exchange trajectory. The value of $n_{\mathbb{R}}$ obtained in section 5.2 is compared with the similarly defi ned parameter obtained in [3] after dividing the latter by the factor $1.23 \pm 0.16$ (equation 11) to account for the different $M_{Y}$ ranges of the two measurements. Since all other parameters describing the sub-leading trajectory are fi xed to the same values in the two analyses, the ratio of $n_{\mathbb{R}}$ results is then equivalent to the ratio of sub-leading exchange contributions in the two cross section measurements. The dominant model dependence uncertainties largely cancel when forming this ratio, which is

$$
\frac{\sigma_{\mathbb{R}}(\mathrm{LRG})}{\sigma_{\mathbb{R}}(\mathrm{FPS})}=1.39 \pm 0.48 \text { (exp.) } \pm 0.29 \text { (model) }
$$

where the fi rst error is the combined statistical and experimental systematic uncertainty and the second is the residual model dependence uncertainty as defi ned in section 5.2. This result is consistent with unity, as expected for a dominantly isosinglet sub-leading trajectory ( $\omega$ or $f$, rather than $\rho$ or $a$ exchanges). It is thus consistent with the conclusion from charge exchange cross section measurements obtained by tagging leading neutrons in DIS at $x_{\mathbb{P}}=0.1$, which can be fully attributed to $\pi$ exchange [7].

The predictions of the '2006 DPDF Fit A' to the H1 LRG data [3] are compared with the FPS data in fi gures 5-7 after applying a factor of $1 / 1.39$ (equation 12) to the sub-leading exchange contribution in the fi $t$ and an overall normalisation factor of $1 / 1.23$ (equation 11) to account for the absence of the proton dissociation contribution in the FPS case. The FPS data are then well described in the region covered by the fit to the LRG data $\left(Q^{2} \geq 8.5 \mathrm{GeV}^{2}\right)$. Extrapolating to lower $Q^{2}$, the description remains reasonable.

The good agreement, after accounting for proton dissociation, between the LRG and the FPS data confi rms that the two measurement methods lead to compatible results, despite having very different systematics. The lack of any kinematic dependence of the ratio of the two 
cross sections shows, within uncertainties, that proton dissociation with $M_{Y}<1.6 \mathrm{GeV}$ can be treated similarly to the elastic proton case. This supports the factorisation, for both the pomeron and the sub-leading exchange contributions, of processes occuring at the proton vertex from those describing the hard interaction, in terms of $M_{Y}$ as well as $t$ (section 5.1) and $x_{\mathbb{P}}$ (section 5.2). It also confi rms that contributions from proton dissociation in the LRG measurement do not signifi cantly alter the measured $\beta, Q^{2}$ or $x_{\mathbb{P}}$ dependences and hence cannot have a large influence on the diffractive gluon density or other information extracted from the LRG data.

\subsection{Comparison with ZEUS leading proton data.}

In fi gure 9 the FPS $\sigma_{r}^{D(3)}$ results are compared with those of the ZEUS collaboration, measured using their Leading Proton Spectrometer (LPS) [5] and also integrated over $|t|<1 \mathrm{GeV}^{2}$. The ZEUS data points are interpolated to the $\beta$ and $Q^{2}$ values of this measurement using the dependences measured in [5]. There is very good agreement between the two data sets. The ratio of the ZEUS LPS to the H1 FPS data averaged over the measured kinematic range is $0.92 \pm 0.04$ (stat.) \pm 0.03 (syst.) \pm 0.15 (norm.), which is consistent with unity taking into account the dominant normalisation uncertainties. Within the errors, there is no $x_{\mathbb{P}}, \beta$ or $Q^{2}$ dependence of the ratio. The sub-leading exchange contributes at a similar level at high $x_{\mathbb{P}}$ and low $\beta$ in both data sets.

\section{Summary}

A semi-inclusive cross section measurement is presented for the diffractive deep-inelastic scattering process $e p \rightarrow e X p$. The results are obtained using data taken with the H1 detector at HERA, where the scattered proton carries at least $90 \%$ of the incoming proton momentum and is measured in the Forward Proton Spectrometer (FPS). The FPS data are in good agreement with those of the ZEUS collaboration obtained with their Leading Proton Spectrometer.

The $t$-dependence is parameterised by an exponential function such that $\mathrm{d} \sigma / \mathrm{d} t \propto e^{B t}$. The resulting values of the slope parameter $B$ in the pomeron dominated range, $x_{\mathbb{P}} \leq 0.0094$, are close to $6 \mathrm{GeV}^{-2}$ and are independent of $x_{\mathbb{P}}$ in this range within errors, favouring an effective pomeron trajectory slope $\alpha_{\mathbb{P}}^{\prime}$ which is close to zero. There is also no signifi cant $Q^{2}$ or $\beta$ dependence of $B$. The slope parameter decreases to around $4 \mathrm{GeV}^{-2}$ in the higher $x_{\mathbb{P}}$ region, where an additional sub-leading exchange is found to contribute.

The diffractive reduced cross section $\sigma_{r}^{D(4)}\left(\beta, Q^{2}, x_{\mathbb{P}}, t\right)$ is measured at $|t|=0.25 \mathrm{GeV}^{2}$. The $x_{\mathbb{P}}$ dependence is described using a model which is motivated by Regge phenomenology, in which a leading pomeron and a sub-leading exchange contribute. The effective pomeron intercept describing the data is $\alpha_{\mathbb{P}}(0)=1.114 \pm 0.018$ (stat.) \pm 0.012 (syst.) ${ }_{-0.020}^{+0.040}$ (model).

The data are also analysed in terms of the diffractive reduced cross section $\sigma_{r}^{D(3)}$, obtained by integrating $\sigma_{r}^{D(4)}$ over the range $\left|t_{\min }\right|<|t|<1 \mathrm{GeV}^{2}$. At fi xed $x_{\mathbb{P}}$, a relatively flat $\beta$ dependence is observed over most of the kinematic range. The data display scaling violations with positive $\partial \sigma_{r}^{D} / \partial \ln Q^{2}$, except at the highest values of $\beta \sim 0.7$. 
The FPS data are compared with the results of an $\mathrm{H} 1$ measurement using events selected on the basis of a large rapidity gap (LRG) rather than a leading proton, which includes proton dissociation to states with masses $M_{Y}<1.6 \mathrm{GeV}$. The ratio of the LRG to the FPS cross section is $1.23 \pm 0.03$ (stat.) \pm 0.16 (syst.), independently of $Q^{2}, \beta$ and $x_{\mathbb{P}}$ within the uncertainties. Apart from this normalisation factor, the FPS and LRG measurements are in remarkably good agreement, despite having very different sources of systematic error. The magnitude of the sub-leading exchange component in the FPS data is compatible with that obtained from the LRG data, suggesting that charge exchange contributions in the latter are small. Within the present uncertainties, the $\mathrm{H} 1$ diffractive DIS data are thus compatible with the factorisation of the variables $x_{\mathbb{P}}, t$ and $M_{Y}$ associated with the proton vertex from the variables $\beta$ and $Q^{2}$, which describe the hard interaction, holding separately for the pomeron and for the sub-leading exchange trajectory.

\section{Acknowledgements}

We are grateful to the HERA machine group whose outstanding efforts have made this experiment possible. We thank the engineers and technicians for their work in constructing and maintaining the $\mathrm{H} 1$ detector, our funding agencies for fi nancial support, the DESY technical staff for continual assistance and the DESY directorate for support and for the hospitality which they extend to the non-DESY members of the collaboration.

\section{References}

[1] T. Ahmed et al. [H1 Collaboration], Nucl. Phys. B 429 (1994) 477;

T. Ahmed et al. [H1 Collaboration], Phys. Lett. B 348 (1995) 681 [hep-ex/9503005].

[2] C. Adloff et al. [H1 Collaboration], Z. Phys. C 76 (1997) 613 [hep-ex/9708016].

[3] H1 Collaboration, "Measurement and QCD Analysis of the Diffractive Deep Inelastic Scattering Cross Section at HERA”, DESY 06-049, submitted to Eur. Phys. J. C.

[4] M. Derrick et al. [ZEUS Collaboration], Phys. Lett. B 315 (1993) 481;

M. Derrick et al. [ZEUS Collaboration], Z. Phys. C 68 (1995) 569 [hep-ex/9505010];

J. Breitweg et al. [ZEUS Collaboration], Eur. Phys. J. C 6 (1999) 43 [hep-ex/9807010];

S. Chekanov et al. [ZEUS Collaboration], Eur. Phys. J. C 25 (2002) 169 [hep-ex/0203039].

[5] S. Chekanov et al. [ZEUS Collaboration], Eur. Phys. J. C 38 (2004) 43 [hep-ex/0408009].

[6] S. Chekanov et al. [ZEUS Collaboration], Nucl. Phys. B 713 (2005) 3 [hep-ex/0501060].

[7] C. Adloff et al. [H1 Collaboration], Eur. Phys. J. C 6 (1999) 587 [hep-ex/9811013].

[8] C. Adloff et al. [H1 Collaboration], Nucl. Phys. B 619 (2001) 3 [hep-ex/0106070].

[9] J. Collins, Phys. Rev. D 57 (1998) 3051 [Erratum-ibid. D 61 (2000) 019902] [hep$\mathrm{ph} / 9709499]$. 
[10] V. Gribov and L. Lipatov, Sov. J. Nucl. Phys. 15 (1972) 438 [Yad. Fiz. 15 (1972) 781]; V. Gribov and L. Lipatov, Sov. J. Nucl. Phys. 15 (1972) 675 [Yad. Fiz. 15 (1972) 1218]; Y. Dokshitzer, Sov. Phys. JETP 46 (1977) 641 [Zh. Eksp. Teor. Fiz. 73 (1977) 1216]; G. Altarelli and G. Parisi, Nucl. Phys. B 126 (1977) 298.

[11] P. Van Esch et al., Nucl. Instrum. Meth. A 446 (2000) 409 [hep-ex/0001046].

[12] I. Abt et al. [H1 Collaboration], Nucl. Instrum. Meth. A 386 (1997) 310; I. Abt et al. [H1 Collaboration], Nucl. Instrum. Meth. A 386 (1997) 348.

[13] R. Appuhn et al. [H1 SPACAL Group], Nucl. Instrum. Meth. A 386 (1997) 397.

[14] C. Adloff et al. [H1 Collaboration], Eur. Phys. J. C 21 (2001) 33 [hep-ex/0012053].

[15] B. Andrieu et al. [H1 Calorimeter Group], Nucl. Instrum. Meth. A 336 (1993) 499; B. Andrieu et al. [H1 Calorimeter Group], Nucl. Instrum. Meth. A 350 (1994) 57.

[16] C. Adloff et al. [H1 Collaboration], Z. Phys. C 74 (1997) 221 [hep-ex/9702003].

[17] O. Karschnik, Ph.D. thesis, University of Hamburg, 2001, available from http://www-h1.desy.de/publications/theses-list.html.

[18] S. Aid et al. [H1 Collaboration], Nucl. Phys. B 463 (1996) 3 [hep-ex/9601004].

[19] S. Bentvelsen et al., Proceedings of the Workshop "Physics at HERA", eds. W. Buchmüller, G. Ingelman, DESY (1992), 23;

C. Hoeger, ibid. 43.

[20] A. Blondel and F. Jacquet, Proceedings of the Study of an $e p$ Facility for Europe, ed. U. Amaldi, DESY 79/48 (1979) 391.

[21] K. Golec-Biernat and M. Wüsthoff, Phys. Rev. D 59 (1999) 014017 [hep-ph/9807513]; K. Golec-Biernat and M. Wüsthoff, Phys. Rev. D 60 (1999) 114023 [hep-ph/9903358].

[22] H. Jung, Comput. Phys. Commun. 86 (1995) 147; (see also http://www.desy.de/ jung/rapgap.html).

[23] B. Andersson, G. Gustafson, G. Ingelman and T. Sjöstrand, Phys. Rept. 97 (1983) 31.

[24] T. Sjöstrand, Comput. Phys. Commun. 82 (1994) 74.

[25] R. Engel and J. Ranft, Phys. Rev. D 54 (1996) 4244 [hep-ph/9509373].

[26] B. List and A. Mastroberardino, Proc. of the Workshop on Monte Carlo Generators for HERA Physics, eds. A. Doyle, G. Grindhammer, G. Ingelman, H. Jung, DESY-PROC1999-02 (1999) 396.

[27] A. Kwiatkowski, H. Spiesberger and H. J. Möhring, Comput. Phys. Commun. 69 (1992) 155. 
[28] G. Jaroszkiewicz and P. Landshoff, Phys. Rev. D 10 (1974) 170;

A. Donnachie and P. Landshoff, Phys. Lett. B 296 (1992) 227 [hep-ph/9209205];

J. Cudell, K. Kang and S. Kim, Phys. Lett. B 395 (1997) 311 [hep-ph/9601336].

[29] S. Chekanov et al. [ZEUS Collaboration], Eur. Phys. J. C 24 (2002) 345 [hep-ex/0201043].

[30] A. Aktas et al. [H1 Collaboration], DESY 05-161, to appear in Eur. Phys. J. C [hepex/0510016].

[31] J. Breitweg et al. [ZEUS Collaboration], Eur. Phys. J. C 14 (2000) 213 [hep-ex/9910038].

[32] J. Bartels and H. Kowalski, Eur. Phys. J. C 19 (2001) 693 [hep-ph/0010345];

I. Ivanov, N. Nikolaev and A. Savin, hep-ph/0501034.

[33] J. Owens, Phys. Rev. D 30 (1984) 943.

[34] W. Apel et al. [Serpukhov-CERN Collaboration], Nucl. Phys. B 154 (1979) 189 [Yad. Fiz. 30 (1979) 373].

[35] A. Kaidalov, Phys. Rept. 50 (1979) 157.

[36] J. Bartels, J. Ellis, H. Kowalski and M. Wüsthoff, Eur. Phys. J. C 7 (1999) 443 [hep$\mathrm{ph} / 9803497]$.

[37] J. Bartels, K. Golec-Biernat and H. Kowalski, Phys. Rev. D 66 (2002) 014001 [hep$\mathrm{ph} / 0203258]$.

[38] A. Hebecker and T. Teubner, Phys. Lett. B 498 (2001) 16 [hep-ph/0010273]. 


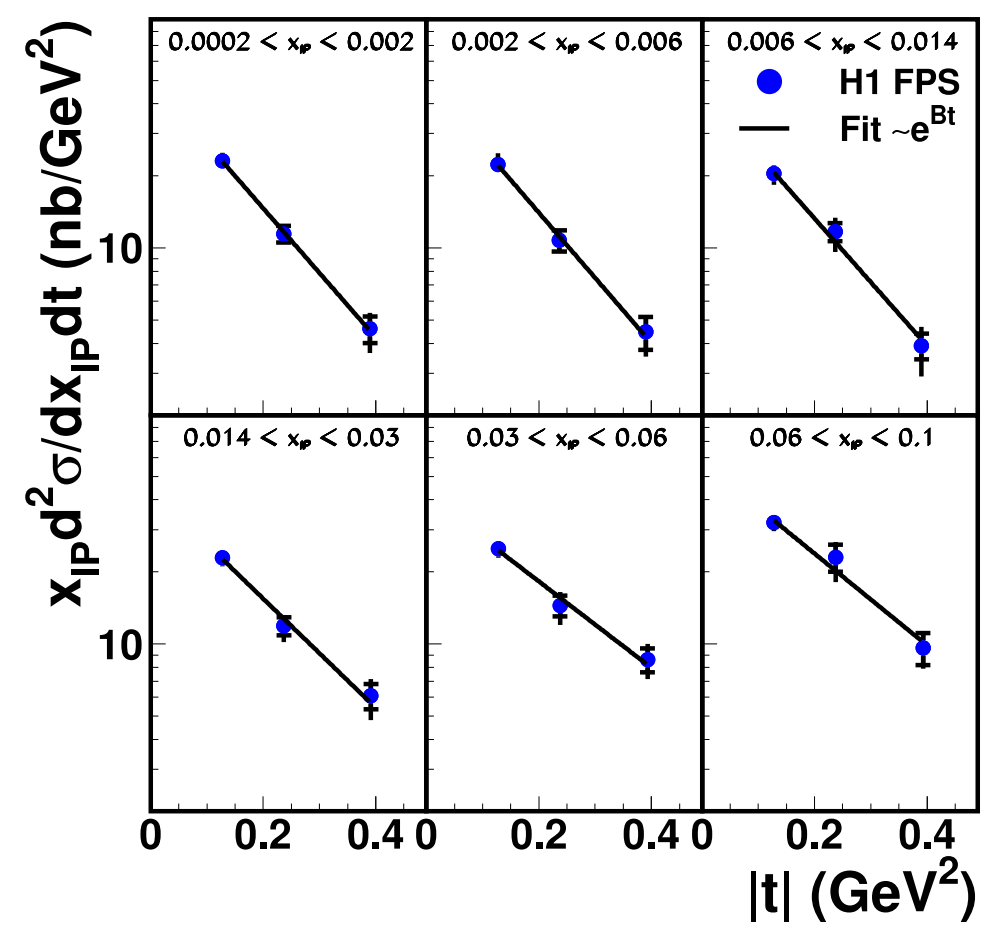

(a)

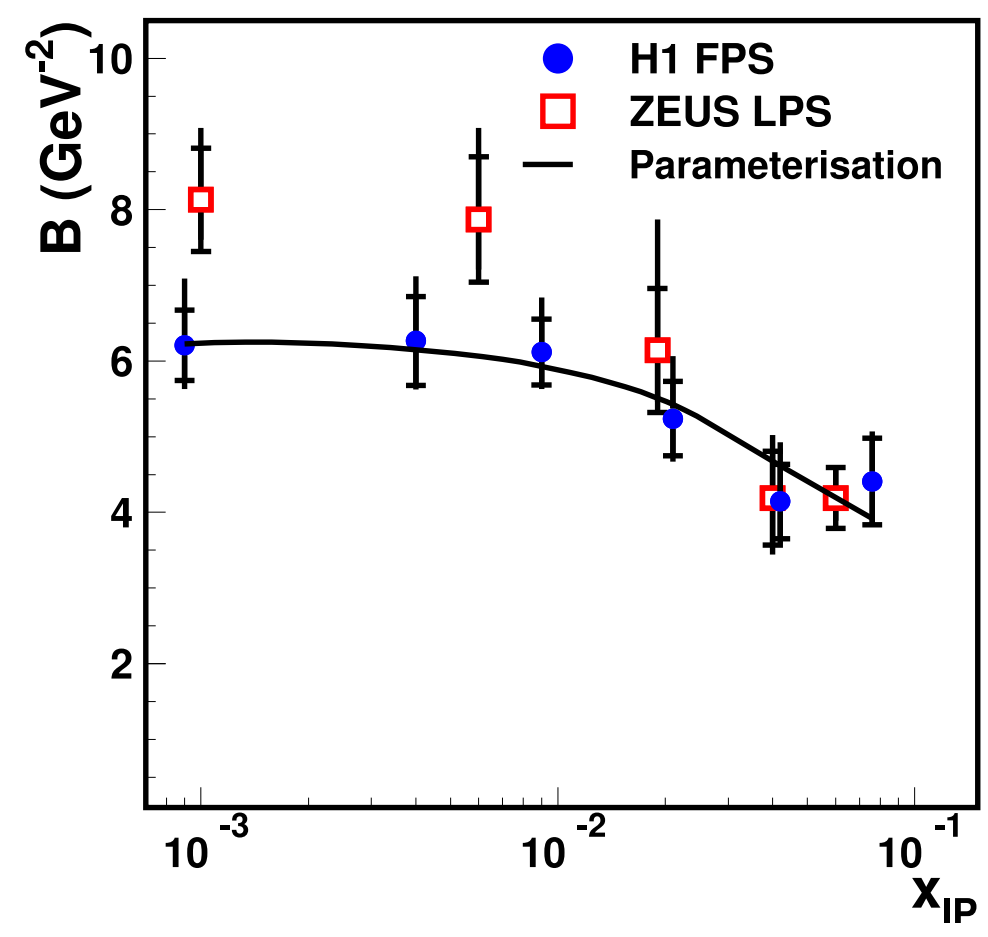

(b)

Figure 2: (a) The differential cross section $x_{\mathbb{P}} \mathrm{d}^{2} \sigma / \mathrm{d} x_{\mathbb{P}} \mathrm{d} t$ measured in the kinematic range $2<Q^{2}<50 \mathrm{GeV}^{2}, 0.02<y<0.6$ for different $x_{\mathbb{P}}$ intervals. The results of $f i$ ts of the form $x_{\mathbb{P}} \mathrm{d}^{2} \sigma / \mathrm{d} x_{\mathbb{P}} \mathrm{d} t \propto e^{B t}$ are also shown. (b) The slope parameter $B$ obtained from these fi ts, shown as a function of $x_{\mathbb{P}}$. The results obtained with the ZEUS LPS [5] and the parameterisation of the H1 data described in section 5.2 are also shown. The inner error bars represent the statistical errors and the outer error bars indicate the statistical and systematic errors added in quadrature. 
(a)

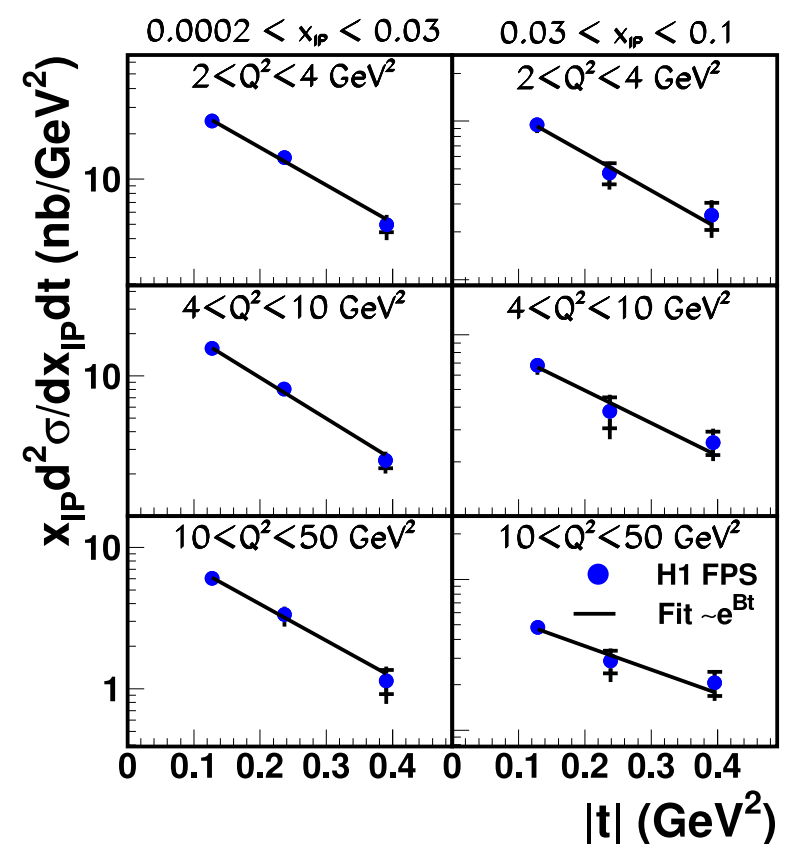

(c)

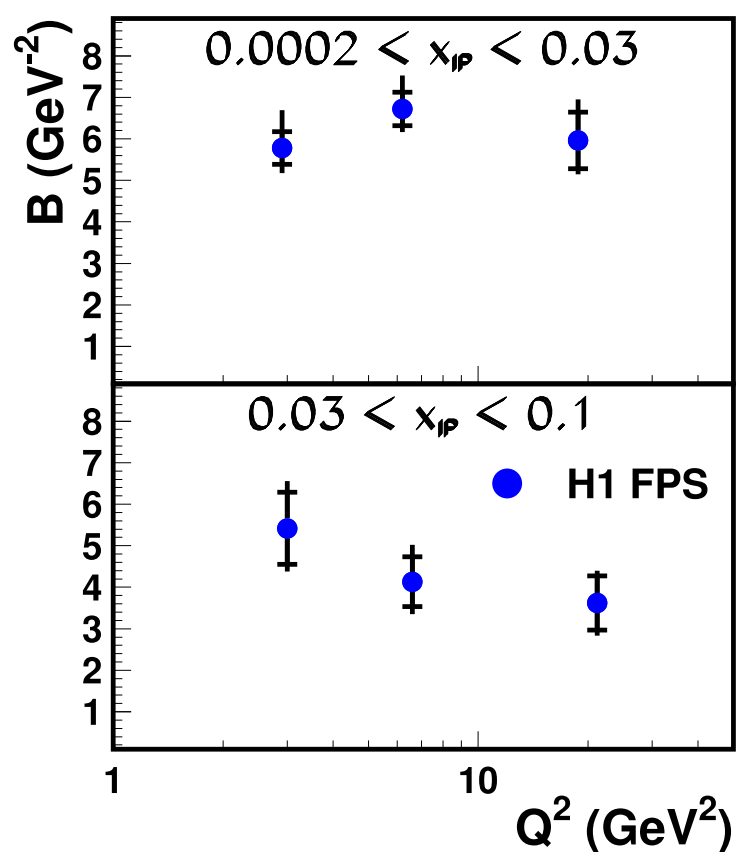

(b)

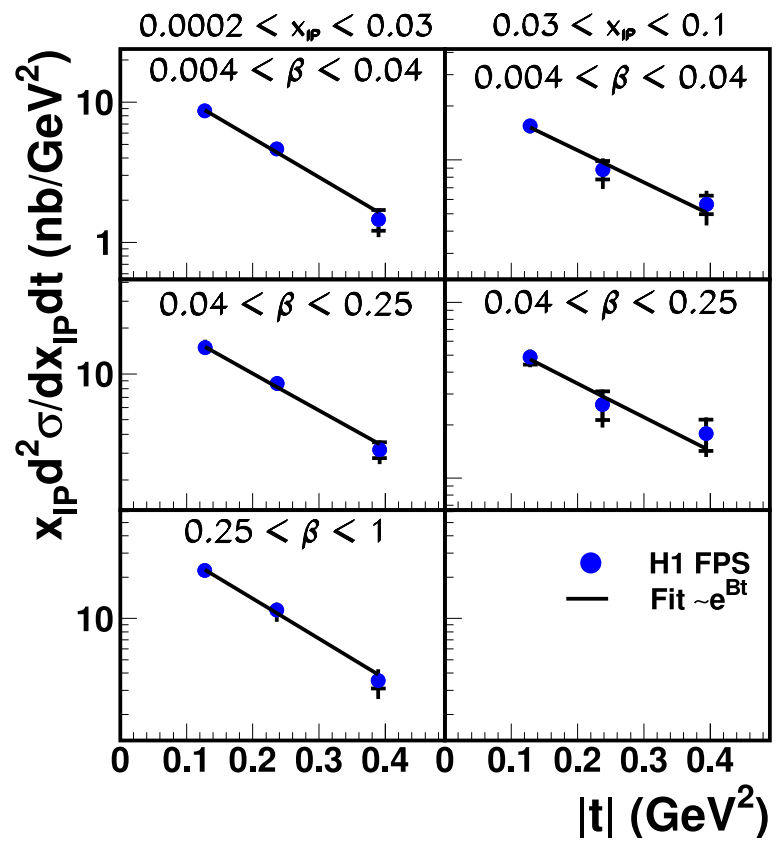

(d)

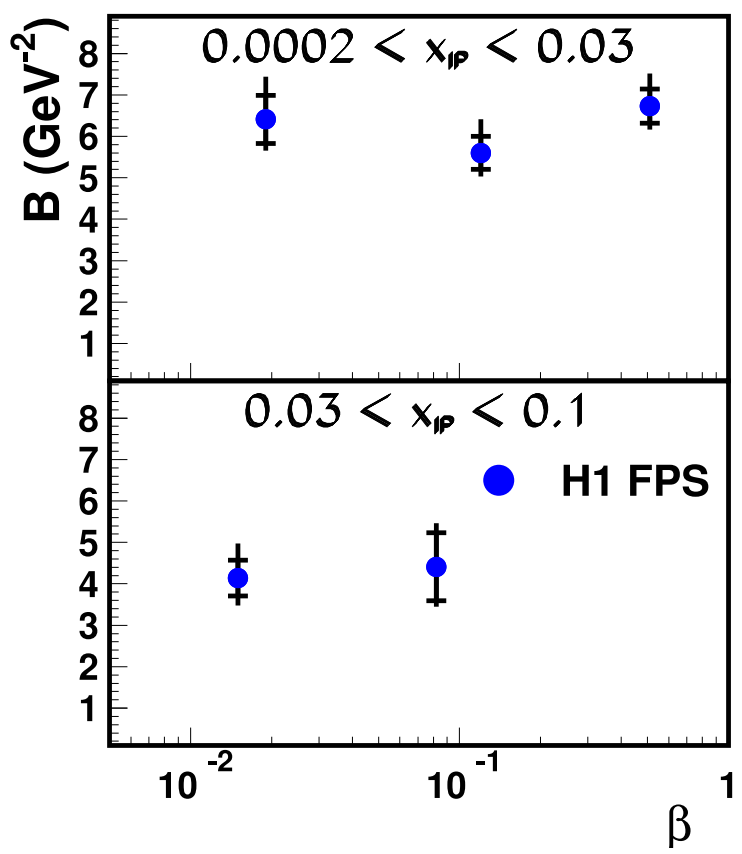

Figure 3: (a,b) The differential cross section $x_{\mathbb{P}} \mathrm{d}^{2} \sigma / \mathrm{d} x_{\mathbb{P}} \mathrm{d} t$ measured in different regions of (a) $Q^{2}$ and $x_{\mathbb{P}}$ and (b) $\beta$ and $x_{\mathbb{P}}$. The results of fi ts of the form $x_{\mathbb{P}} \mathrm{d}^{2} \sigma / \mathrm{d} x_{\mathbb{P}} \mathrm{d} t \propto e^{B t}$ are shown. (c,d) The slope parameter $B$ obtained from these fi ts, shown as a function of (c) $Q^{2}$ and (d) $\beta$ for two $x_{\mathbb{P}}$ intervals. The inner error bars represent the statistical errors and the outer error bars indicate the statistical and systematic errors added in quadrature. 


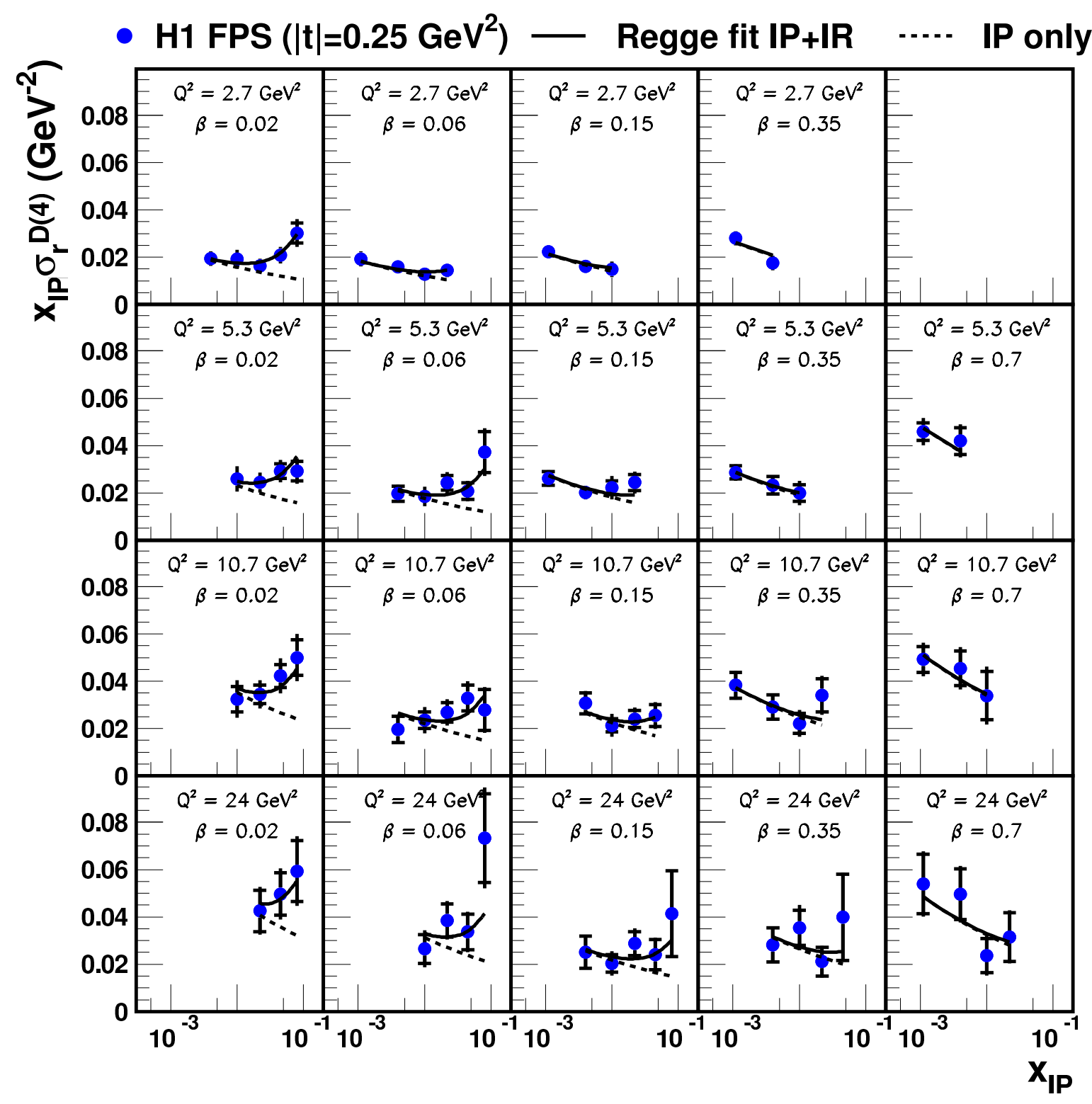

Figure 4: The diffractive reduced cross section $x_{\mathbb{P}} \sigma_{r}^{D(4)}\left(\beta, Q^{2}, x_{\mathbb{P}}, t\right)$, shown as a function of $x_{\mathbb{P}}$ for $|t|=0.25 \mathrm{GeV}^{2}$ at different values of $\beta$ and $Q^{2}$. The inner error bars represent the statistical errors. The outer error bars indicate the statistical and systematic errors added in quadrature. An overall normalisation uncertainty of $10.1 \%$ is not shown. The solid curves represent the results of the phenomenological 'Regge' fi t to the data, including both pomeron $(\mathbb{P})$ and sub-leading $(\mathbb{R})$ trajectory exchange, as described in section 5.2. The dashed curves represent the contribution from pomeron exchange alone according to the fi t. 


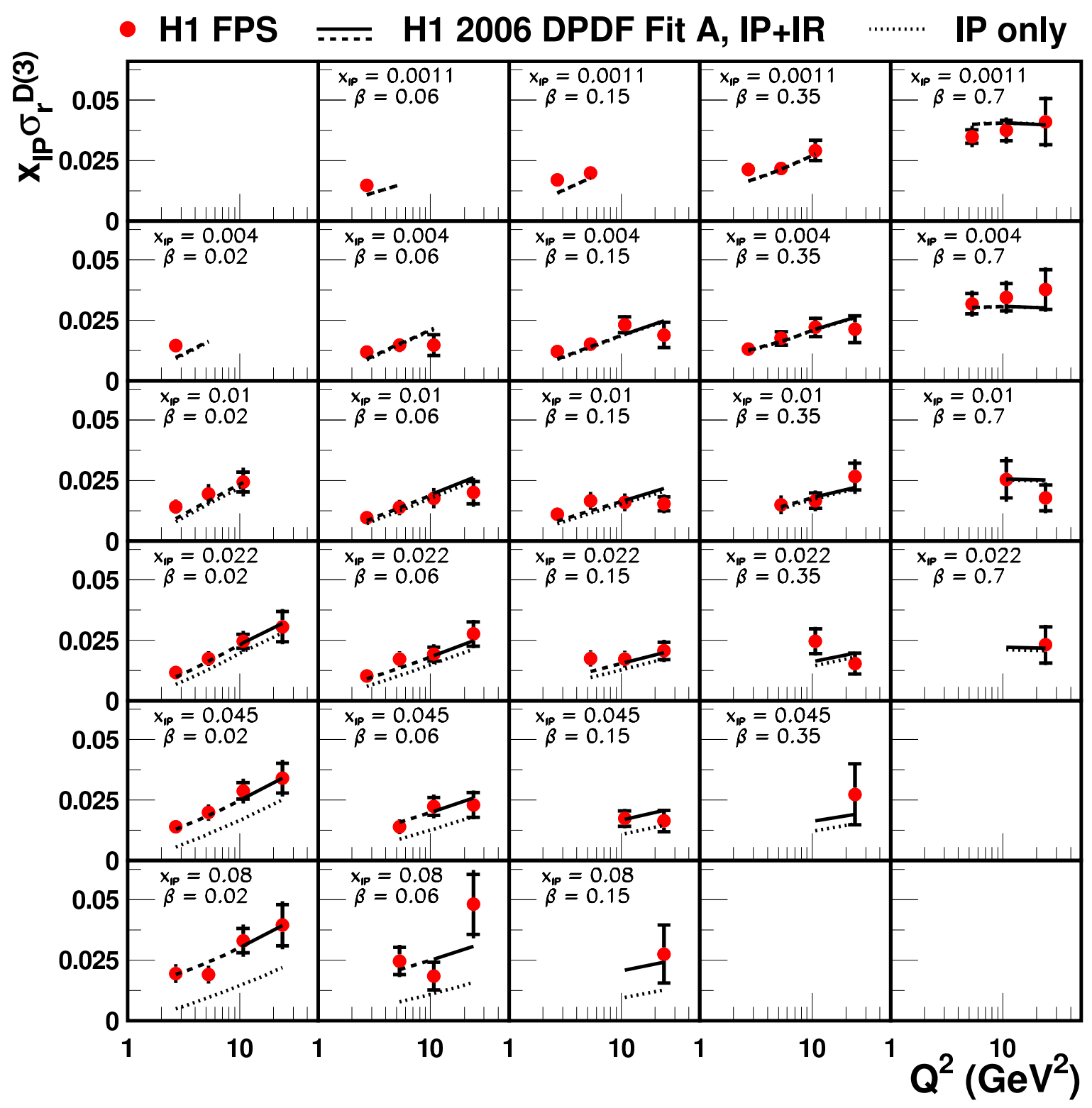

Figure 5: The diffractive reduced cross section $x_{\mathbb{P}} \sigma_{r}^{D(3)}\left(\beta, Q^{2}, x_{\mathbb{P}}\right)$ for $|t|<1 \mathrm{GeV}^{2}$, shown as a function of $Q^{2}$ for different values of $x_{\mathbb{P}}$ and $\beta$. The inner error bars represent the statistical errors. The outer error bars indicate the statistical and systematic errors added in quadrature. An overall normalisation uncertainty of $10.1 \%$ is not shown. The solid curves represent the results of the 'H1 2006 DPDF Fit A' to LRG data [3], modifi ed as described in section 6.1. The dashed curves represent the extrapolation of this prediction beyond the $Q^{2}$ range which is included in the fi t. The dotted curves indicate the contribution of pomeron exchange alone in this model. 


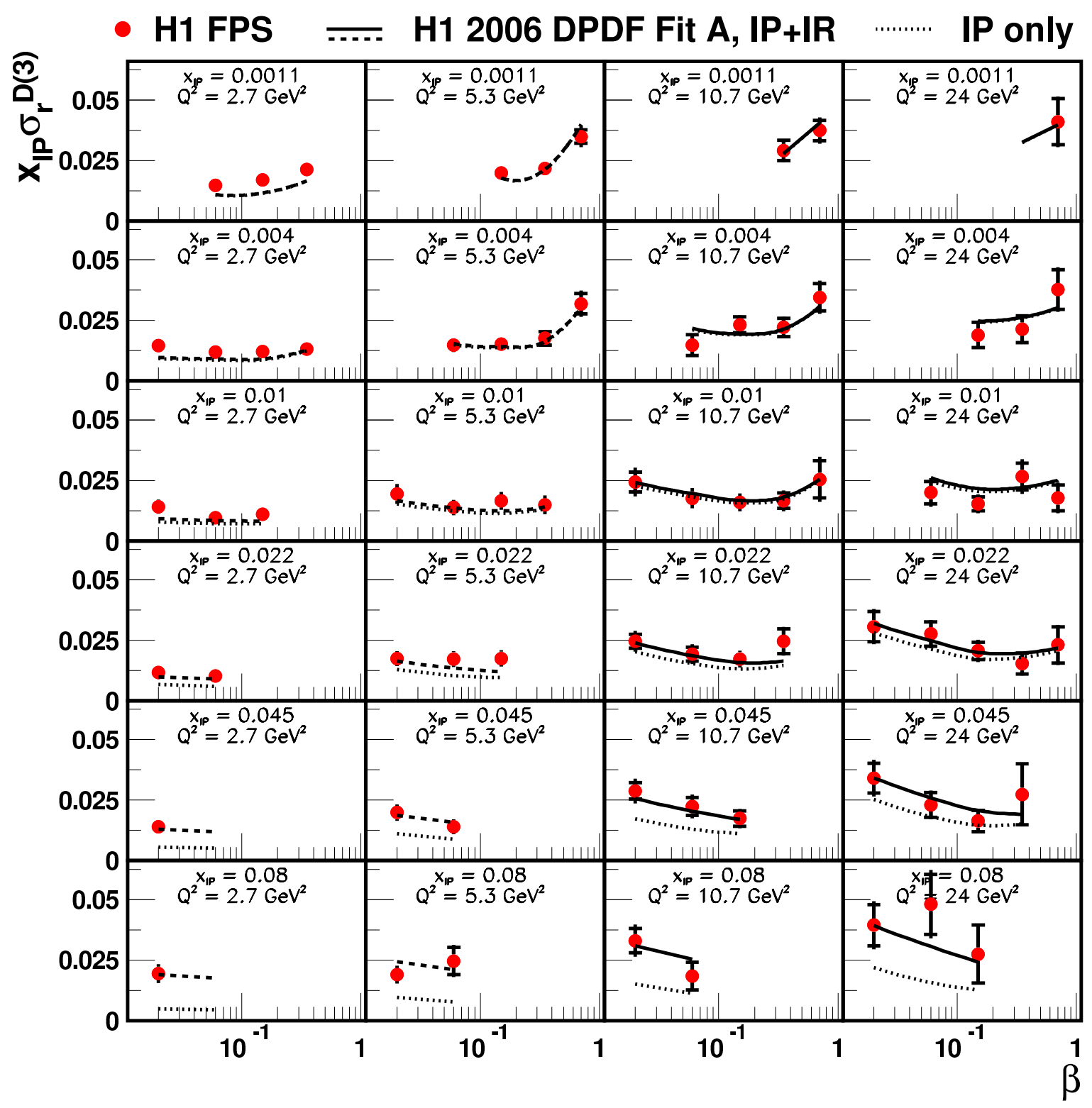

Figure 6: The diffractive reduced cross section $x_{\mathbb{P}} \sigma_{r}^{D(3)}\left(\beta, Q^{2}, x_{\mathbb{P}}\right)$ for $|t|<1 \mathrm{GeV}^{2}$, shown as a function of $\beta$ for different values of $x_{\mathbb{P}}$ and $Q^{2}$. The inner error bars represent the statistical errors. The outer error bars indicate the statistical and systematic errors added in quadrature. An overall normalisation uncertainty of $10.1 \%$ is not shown. The solid curves represent the results of the 'H1 2006 DPDF Fit A' to LRG data [3], modifi ed as described in section 6.1. The dashed curves represent the extrapolation of this prediction beyond the $Q^{2}$ range which is included in the fi t. The dotted curves indicate the contribution of pomeron exchange alone in this model. 


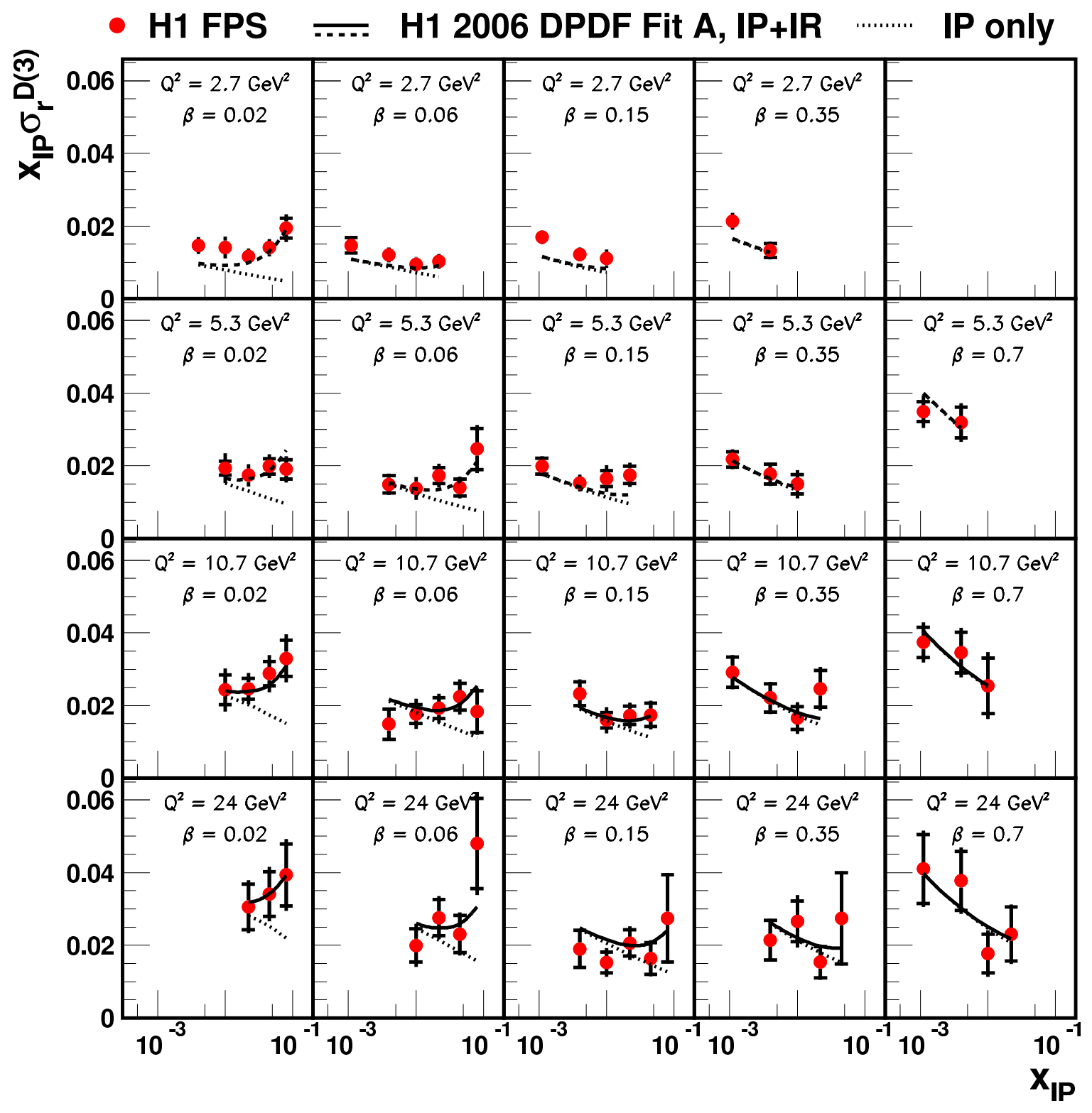

Figure 7: The diffractive reduced cross section $x_{\mathbb{P}} \sigma_{r}^{D(3)}\left(\beta, Q^{2}, x_{\mathbb{P}}\right)$ for $|t|<1 \mathrm{GeV}^{2}$, shown as a function of $x_{\mathbb{P}}$ for different values of $\beta$ and $Q^{2}$. The inner error bars represent the statistical errors. The outer error bars indicate the statistical and systematic errors added in quadrature. An overall normalisation uncertainty of $10.1 \%$ is not shown. The solid curves represent the results of the 'H1 2006 DPDF Fit A' to LRG data [3], modifi ed as described in section 6.1. The dashed curves represent the extrapolation of this prediction beyond the $Q^{2}$ range which is included in the fi t. The dotted curves indicate the contribution of pomeron exchange alone in this model. 
(a)

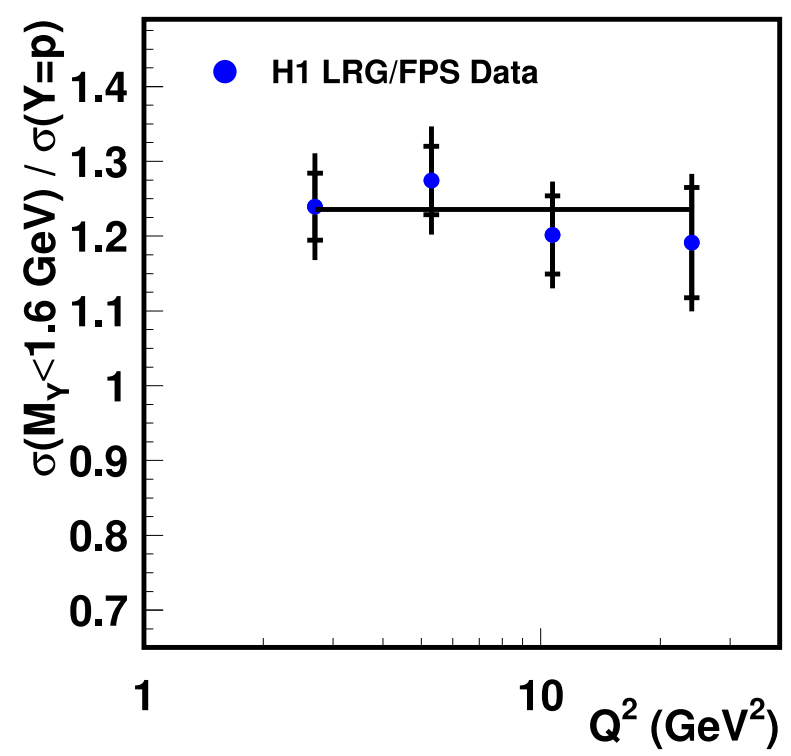

(b)

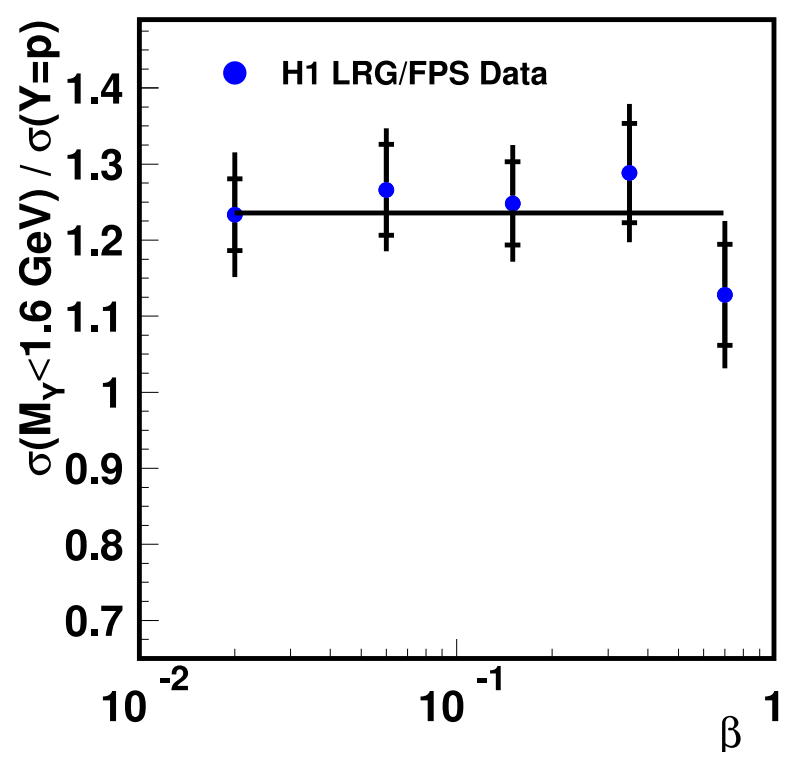

(c)

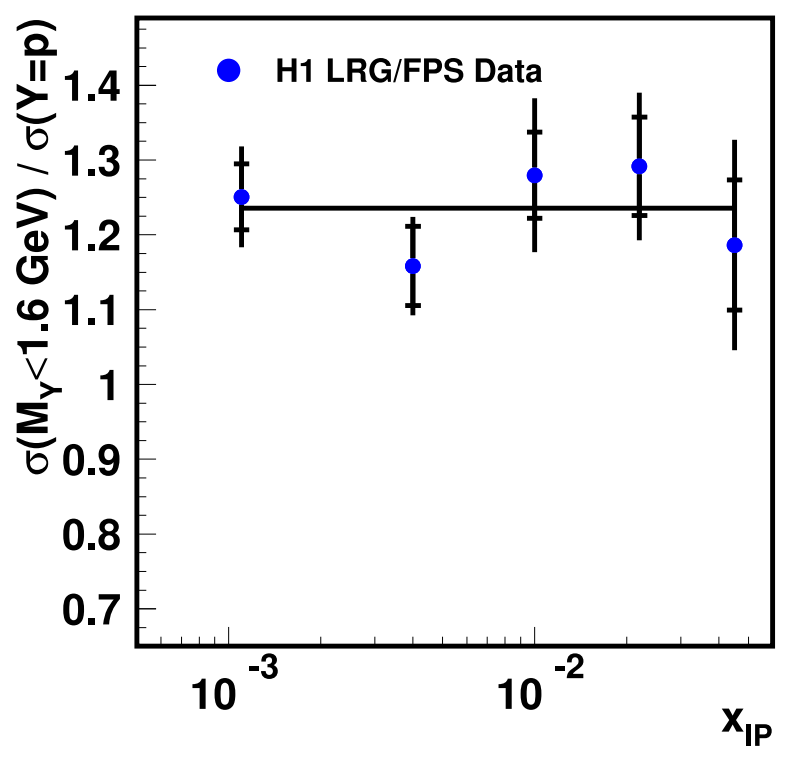

Figure 8: The ratio of the diffractive cross section for $M_{Y}<1.6 \mathrm{GeV}$ and $|t|<1 \mathrm{GeV}^{2}$ to that for $Y=p$ and $|t|<1 \mathrm{GeV}^{2}$, obtained from $\sigma_{r}^{D(3)}$ measurements using the LRG and FPS methods. The results are shown as a function of (a) $Q^{2}$, (b) $\beta$ and (c) $x_{\mathbb{P}}$, after averaging over the other variables. The lines represent the result of a fi $t$ to the data assuming no dependence on any of these variables. The inner error bars represent the statistical errors. The outer error bars indicate the statistical and systematic errors added in quadrature. Normalisation uncertainties of $12.7 \%$ are not shown. 
- H1 FPS $\triangle$ ZEUS LPS

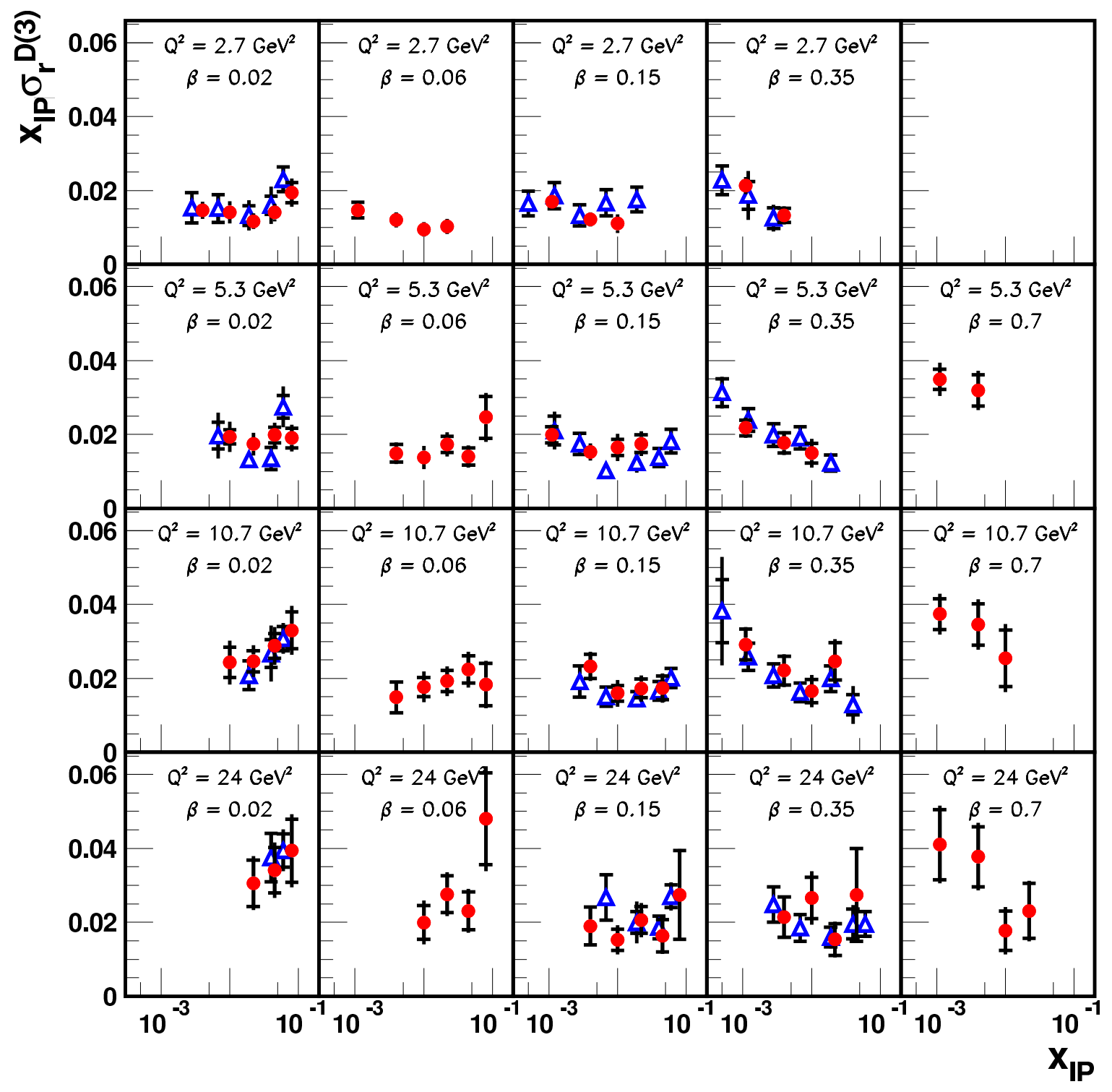

Figure 9: The diffractive reduced cross section $x_{\mathbb{P}} \sigma_{r}^{D(3)}\left(\beta, Q^{2}, x_{\mathbb{P}}\right)$ for $|t|<1 \mathrm{GeV}^{2}$, shown as a function of $x_{\mathbb{P}}$ for different values of $\beta$ and $Q^{2}$. H1 FPS data are compared with ZEUS LPS results [5]. The inner error bars represent the statistical errors. The outer error bars indicate the statistical and systematic errors added in quadrature. Normalisation uncertainties of around $10 \%$ on each data set are not shown. 


\begin{tabular}{|c|c|c|c|c|c|c|}
\hline$Q^{2}$ bin $\left[\mathrm{GeV}^{2}\right]$ & $\left\langle Q^{2}\right\rangle\left[\mathrm{GeV}^{2}\right]$ & $\beta$ bin & $\langle\beta\rangle$ & $x_{\mathbb{P}}$ bin & $\left\langle x_{\mathbb{P}}\right\rangle$ & $B\left[\mathrm{GeV}^{-2}\right]$ \\
\hline $2-50$ & 5.4 & $0.004-1$ & 0.4 & $0.0002-0.002$ & 0.0009 & $6.21 \pm 0.46_{-0.35}^{+0.75}$ \\
$2-50$ & 7.5 & $0.004-1$ & 0.23 & $0.002-0.006$ & 0.0036 & $6.26 \pm 0.59_{-0.25}^{+0.62}$ \\
$2-50$ & 7.9 & $0.004-1$ & 0.1 & $0.006-0.014$ & 0.0094 & $6.14 \pm 0.44_{-0.22}^{+0.58}$ \\
$2-50$ & 9.0 & $0.004-1$ & 0.06 & $0.014-0.03$ & 0.021 & $5.36 \pm 0.53_{-0.28}^{+0.66}$ \\
$2-50$ & 10.3 & $0.004-1$ & 0.037 & $0.03-0.06$ & 0.042 & $4.16 \pm 0.50_{-0.26}^{+0.61}$ \\
$2-50$ & 12.1 & $0.004-1$ & 0.023 & $0.06-0.1$ & 0.076 & $4.48 \pm 0.56_{-0.07}^{+0.33}$ \\
\hline
\end{tabular}

Table 3: The slope parameter $B$, extracted from fi ts to the data of the form $\mathrm{d} \sigma / \mathrm{d} t \propto e^{B t}$ in different regions of $x_{\mathbb{P}}$. The mean values of $Q^{2}, \beta$ and $x_{\mathbb{P}}$ are also shown for each measurement. The fi rst uncertainty given is statistical, the second systematic.

\begin{tabular}{|c|c|c|c|c|c|c|}
\hline$Q^{2}$ bin $\left[\mathrm{GeV}^{2}\right]$ & $\left\langle Q^{2}\right\rangle\left[\mathrm{GeV}^{2}\right]$ & $\beta$ bin & $\langle\beta\rangle$ & $x_{\mathbb{P}}$ bin & $\left\langle x_{\mathbb{P}}\right\rangle$ & $B\left[\mathrm{GeV}^{-2}\right]$ \\
\hline $2-50$ & 5.1 & $0.004-0.04$ & 0.019 & $0.0002-0.03$ & 0.013 & $6.41 \pm 0.58_{-0.48}^{+0.85}$ \\
$2-50$ & 9.1 & $0.004-0.04$ & 0.015 & $0.03-0.1$ & 0.054 & $4.14 \pm 0.43_{-0.51}^{+0.72}$ \\
$2-50$ & 7.9 & $0.04-0.25$ & 0.12 & $0.0002-0.03$ & 0.0074 & $5.60 \pm 0.40_{-0.41}^{+0.71}$ \\
$2-50$ & 16.3 & $0.04-0.25$ & 0.082 & $0.03-0.1$ & 0.048 & $4.41 \pm 0.82_{-0.51}^{+0.66}$ \\
$2-50$ & 8.4 & $0.25-1$ & 0.51 & $0.0002-0.03$ & 0.0027 & $6.73 \pm 0.41_{-0.39}^{+0.67}$ \\
$2-4$ & 2.9 & $0.004-1$ & 0.19 & $0.0002-0.03$ & 0.0065 & $5.78 \pm 0.39_{-0.47}^{+0.83}$ \\
$2-4$ & 3.0 & $0.004-1$ & 0.016 & $0.03-0.1$ & 0.051 & $5.42 \pm 0.87_{-0.57}^{+0.73}$ \\
$4-10$ & 6.2 & $0.004-1$ & 0.23 & $0.0002-0.03$ & 0.0077 & $6.72 \pm 0.40_{-0.39}^{+0.70}$ \\
$4-10$ & 6.6 & $0.004-1$ & 0.024 & $0.03-0.1$ & 0.052 & $4.13 \pm 0.60_{-0.49}^{+0.66}$ \\
$10-50$ & 18.8 & $0.004-1$ & 0.26 & $0.0002-0.03$ & 0.01 & $5.96 \pm 0.68_{-0.44}^{+0.72}$ \\
$10-50$ & 21.2 & $0.004-1$ & 0.054 & $0.03-0.1$ & 0.055 & $3.62 \pm 0.65_{-0.44}^{+0.43}$ \\
\hline
\end{tabular}

Table 4: The slope parameter $B$ extracted from fi ts to the data of the form $\mathrm{d} \sigma / \mathrm{d} t \propto e^{B t}$ in different regions of $x_{\mathbb{P}}, \beta$ and $Q^{2}$. The mean values of these kinematic variables are also given for each measurement. The fi rst uncertainty given is statistical, the second systematic. 


\begin{tabular}{|c|c|c|c|c|}
\hline$Q^{2}\left[\mathrm{GeV}^{2}\right]$ & $\beta$ & $x_{\mathbb{P}}$ & $x_{\mathbb{P}} \sigma_{r}^{D(4)}\left[\mathrm{GeV}^{-2}\right]$ & $x_{\mathbb{P}} \sigma_{r}^{D(3)}$ \\
\hline 2.7 & 0.02 & 0.0040 & $0.0194 \pm 0.0023_{-0.0013}^{+0.0027}$ & $0.0147 \pm 0.0017_{-0.0010}^{+0.0021}$ \\
2.7 & 0.02 & 0.0100 & $0.0193 \pm 0.0016_{-0.0041}^{+0.0033}$ & $0.0141 \pm 0.0012_{-0.0030}^{+0.0025}$ \\
2.7 & 0.02 & 0.0220 & $0.0163 \pm 0.0016_{-0.0028}^{+0.0021}$ & $0.0116 \pm 0.0011_{-0.0020}^{+0.0016}$ \\
2.7 & 0.02 & 0.0450 & $0.0209 \pm 0.0022_{-0.0031}^{+0.0025}$ & $0.0140 \pm 0.0015_{-0.0021}^{+0.0018}$ \\
2.7 & 0.02 & 0.0800 & $0.0306 \pm 0.0042_{-0.0047}^{+0.0036}$ & $0.0195 \pm 0.0027_{-0.0030}^{+0.0024}$ \\
2.7 & 0.06 & 0.0011 & $0.0192 \pm 0.0027_{-0.0010}^{+0.0018}$ & $0.0147 \pm 0.0021_{-0.0008}^{+0.0015}$ \\
2.7 & 0.06 & 0.0040 & $0.0159 \pm 0.0022_{-0.0009}^{+0.0020}$ & $0.0120 \pm 0.0016_{-0.0007}^{+0.0016}$ \\
2.7 & 0.06 & 0.0100 & $0.0129 \pm 0.0014_{-0.0025}^{+0.0020}$ & $0.0095 \pm 0.0011_{-0.0019}^{+0.0015}$ \\
2.7 & 0.06 & 0.0220 & $0.0145 \pm 0.0021_{-0.0022}^{+0.0017}$ & $0.0103 \pm 0.0015_{-0.0016}^{+0.0012}$ \\
2.7 & 0.15 & 0.0011 & $0.0224 \pm 0.0017_{-0.0012}^{+0.0022}$ & $0.0170 \pm 0.0013_{-0.0009}^{+0.0018}$ \\
2.7 & 0.15 & 0.0040 & $0.0161 \pm 0.0019_{-0.0009}^{+0.0020}$ & $0.0122 \pm 0.0014_{-0.0007}^{+0.0016}$ \\
2.7 & 0.15 & 0.0100 & $0.0149 \pm 0.0019_{-0.0029}^{+0.0026}$ & $0.0110 \pm 0.0014_{-0.0022}^{+0.0020}$ \\
2.7 & 0.35 & 0.0011 & $0.0279 \pm 0.0021_{-0.0015}^{+0.0027}$ & $0.0213 \pm 0.0016_{-0.0012}^{+0.0022}$ \\
2.7 & 0.35 & 0.0040 & $0.0177 \pm 0.0025_{-0.0012}^{+0.0026}$ & $0.0133 \pm 0.0019_{-0.0009}^{+0.0020}$ \\
\hline
\end{tabular}

Table 5: The diffractive reduced cross sections, $x_{\mathbb{P}} \sigma_{r}^{D(4)}$ measured at $|t|=0.25 \mathrm{GeV}^{2}$, and $x_{\mathbb{P}} \sigma_{r}^{D(3)}$ integrated over $\left|t_{\text {min }}\right|<|t|<1 \mathrm{GeV}^{2}$, measured at $Q^{2}=2.7 \mathrm{GeV}^{2}$ and various $\beta$ and $x_{\mathbb{P}}$ values. The fi rst uncertainty given is statistical, the second systematic. Normalisation uncertainties of $10.1 \%$ are not included. 


\begin{tabular}{|c|c|c|c|c|}
\hline$Q^{2}\left[\mathrm{GeV}^{2}\right]$ & $\beta$ & $x_{\mathbb{P}}$ & $x_{\mathbb{P}} \sigma_{r}^{D(4)}\left[\mathrm{GeV}^{-2}\right]$ & $x_{\mathbb{P}} \sigma_{r}^{D(3)}$ \\
\hline 5.3 & 0.02 & 0.0100 & $0.0258 \pm 0.0025_{-0.0053}^{+0.0041}$ & $0.0194 \pm 0.0019_{-0.0040}^{+0.0031}$ \\
5.3 & 0.02 & 0.0220 & $0.0243 \pm 0.0023_{-0.0041}^{+0.0029}$ & $0.0174 \pm 0.0017_{-0.0030}^{+0.0022}$ \\
5.3 & 0.02 & 0.0450 & $0.0290 \pm 0.0031_{-0.0042}^{+0.0030}$ & $0.0199 \pm 0.0021_{-0.0029}^{+0.0022}$ \\
5.3 & 0.02 & 0.0800 & $0.0295 \pm 0.0040_{-0.0043}^{+0.0031}$ & $0.0190 \pm 0.0027_{-0.0028}^{+0.0021}$ \\
5.3 & 0.06 & 0.0040 & $0.0197 \pm 0.0032_{-0.0014}^{+0.0025}$ & $0.0149 \pm 0.0024_{-0.0011}^{+0.0019}$ \\
5.3 & 0.06 & 0.0100 & $0.0185 \pm 0.0023_{-0.0039}^{+0.0030}$ & $0.0138 \pm 0.0017_{-0.0029}^{+0.0023}$ \\
5.3 & 0.06 & 0.0220 & $0.0240 \pm 0.0031_{-0.0039}^{+0.0028}$ & $0.0173 \pm 0.0022_{-0.0028}^{+0.0021}$ \\
5.3 & 0.06 & 0.0450 & $0.0208 \pm 0.0034_{-0.0031}^{+0.0022}$ & $0.0140 \pm 0.0023_{-0.0021}^{+0.0016}$ \\
5.3 & 0.06 & 0.0800 & $0.0369 \pm 0.0087_{-0.0060}^{+0.0041}$ & $0.0246 \pm 0.0057_{-0.0041}^{+0.0028}$ \\
5.3 & 0.15 & 0.0011 & $0.0260 \pm 0.0029_{-0.0015}^{+0.0024}$ & $0.0199 \pm 0.0022_{-0.0012}^{+0.0020}$ \\
5.3 & 0.15 & 0.0040 & $0.0202 \pm 0.0021_{-0.0012}^{+0.0027}$ & $0.0153 \pm 0.0016_{-0.0010}^{+0.0001}$ \\
5.3 & 0.15 & 0.0100 & $0.0222 \pm 0.0030_{-0.0044}^{+0.0035}$ & $0.0165 \pm 0.0022_{-0.0033}^{+0.0026}$ \\
5.3 & 0.15 & 0.0220 & $0.0243 \pm 0.0034_{-0.0037}^{+0.0027}$ & $0.0175 \pm 0.0024_{-0.0026}^{+0.0020}$ \\
5.3 & 0.35 & 0.0011 & $0.0286 \pm 0.0028_{-0.0019}^{+0.0025}$ & $0.0218 \pm 0.0021_{-0.0015}^{+0.0020}$ \\
5.3 & 0.35 & 0.0040 & $0.0232 \pm 0.0036_{-0.0015}^{+0.0027}$ & $0.0177 \pm 0.0027_{-0.0012}^{+0.0021}$ \\
5.3 & 0.35 & 0.0100 & $0.0200 \pm 0.0036_{-0.0041}^{+0.0035}$ & $0.0149 \pm 0.0027_{-0.0031}^{+0.0027}$ \\
5.3 & 0.70 & 0.0011 & $0.0460 \pm 0.0037_{-0.0040}^{+0.0049}$ & $0.0349 \pm 0.0028_{-0.0031}^{+0.0039}$ \\
5.3 & 0.70 & 0.0040 & $0.0419 \pm 0.0056_{-0.0033}^{+0.0049}$ & $0.0319 \pm 0.0043_{-0.0026}^{+0.0039}$ \\
\hline
\end{tabular}

Table 6: The diffractive reduced cross sections, $x_{\mathbb{P}} \sigma_{r}^{D(4)}$ measured at $|t|=0.25 \mathrm{GeV}^{2}$, and $x_{\mathbb{P}} \sigma_{r}^{D(3)}$ integrated over $\left|t_{\text {min }}\right|<|t|<1 \mathrm{GeV}^{2}$, measured at $Q^{2}=5.3 \mathrm{GeV}^{2}$ and various $\beta$ and $x_{\mathbb{P}}$ values. The fi rst uncertainty given is statistical, the second systematic. Normalisation uncertainties of $10.1 \%$ are not included. 


\begin{tabular}{|c|c|c|c|c|}
\hline$Q^{2}\left[\mathrm{GeV}^{2}\right]$ & $\beta$ & $x_{\mathbb{P}}$ & $x_{\mathbb{P}} \sigma_{r}^{D(4)}\left[\mathrm{GeV}^{-2}\right]$ & $x_{\mathbb{P}} \sigma_{r}^{D(3)}$ \\
\hline 10.7 & 0.02 & 0.0100 & $0.0325 \pm 0.0054_{-0.0064}^{+0.0050}$ & $0.0243 \pm 0.0040_{-0.0048}^{+0.0039}$ \\
10.7 & 0.02 & 0.0220 & $0.0345 \pm 0.0040_{-0.0050}^{+0.0037}$ & $0.0246 \pm 0.0028_{-0.0036}^{+0.0027}$ \\
10.7 & 0.02 & 0.0450 & $0.0422 \pm 0.0049_{-0.0064}^{+0.0047}$ & $0.0288 \pm 0.0033_{-0.0044}^{+0.0034}$ \\
10.7 & 0.02 & 0.0800 & $0.0502 \pm 0.0075_{-0.0076}^{+0.0053}$ & $0.0330 \pm 0.0050_{-0.0051}^{+0.0036}$ \\
10.7 & 0.06 & 0.0040 & $0.0196 \pm 0.0056_{-0.0012}^{+0.0024}$ & $0.0149 \pm 0.0042_{-0.0009}^{+0.0019}$ \\
10.7 & 0.06 & 0.0100 & $0.0236 \pm 0.0034_{-0.0048}^{+0.0038}$ & $0.0177 \pm 0.0026_{-0.0036}^{+0.0029}$ \\
10.7 & 0.06 & 0.0220 & $0.0269 \pm 0.0041_{-0.0044}^{+0.0032}$ & $0.0193 \pm 0.0029_{-0.0032}^{+0.0023}$ \\
10.7 & 0.06 & 0.0450 & $0.0329 \pm 0.0054_{-0.0053}^{+0.0038}$ & $0.0224 \pm 0.0037_{-0.0037}^{+0.0027}$ \\
10.7 & 0.06 & 0.0800 & $0.0278 \pm 0.0087_{-0.0051}^{+0.0033}$ & $0.0184 \pm 0.0057_{-0.0034}^{+0.0023}$ \\
10.7 & 0.15 & 0.0040 & $0.0309 \pm 0.0044_{-0.0020}^{+0.0034}$ & $0.0232 \pm 0.0033_{-0.0016}^{+0.0027}$ \\
10.7 & 0.15 & 0.0100 & $0.0213 \pm 0.0028_{-0.0044}^{+0.0035}$ & $0.0160 \pm 0.0021_{-0.0034}^{+0.0027}$ \\
10.7 & 0.15 & 0.0220 & $0.0240 \pm 0.0036_{-0.0038}^{+0.0028}$ & $0.0173 \pm 0.0026_{-0.0028}^{+0.0020}$ \\
10.7 & 0.15 & 0.0450 & $0.0254 \pm 0.0047_{-0.0036}^{+0.0006}$ & $0.0174 \pm 0.0032_{-0.0025}^{+0.0019}$ \\
10.7 & 0.35 & 0.0011 & $0.0382 \pm 0.0055_{-0.0021}^{+0.0032}$ & $0.0292 \pm 0.0042_{-0.0017}^{+0.0006}$ \\
10.7 & 0.35 & 0.0040 & $0.0292 \pm 0.0051_{-0.0018}^{+0.0035}$ & $0.0221 \pm 0.0039_{-0.0014}^{+0.0027}$ \\
10.7 & 0.35 & 0.0100 & $0.0222 \pm 0.0042_{-0.00043}^{+0.0034}$ & $0.0166 \pm 0.0032_{-0.0032}^{+0.0026}$ \\
10.7 & 0.35 & 0.0220 & $0.0341 \pm 0.0070_{-0.0049}^{+0.0038}$ & $0.0246 \pm 0.0051_{-0.0036}^{+0.0028}$ \\
10.7 & 0.70 & 0.0011 & $0.0492 \pm 0.0055_{-0.0044}^{+0.0047}$ & $0.0374 \pm 0.0041_{-0.0034}^{+0.0038}$ \\
10.7 & 0.70 & 0.0040 & $0.0454 \pm 0.0073_{-0.0040}^{+0.0061}$ & $0.0346 \pm 0.0056_{-0.0031}^{+0.0048}$ \\
10.7 & 0.70 & 0.0100 & $0.0339 \pm 0.0102_{-0.0071}^{+0.0064}$ & $0.0254 \pm 0.0077_{-0.0053}^{+0.0049}$ \\
\hline
\end{tabular}

Table 7: The diffractive reduced cross sections, $x_{\mathbb{P}} \sigma_{r}^{D(4)}$ measured at $|t|=0.25 \mathrm{GeV}^{2}$, and $x_{\mathbb{P}} \sigma_{r}^{D(3)}$ integrated over $\left|t_{\text {min }}\right|<|t|<1 \mathrm{GeV}^{2}$, measured at $Q^{2}=10.7 \mathrm{GeV}^{2}$ and various $\beta$ and $x_{\mathbb{P}}$ values. The fi rst uncertainty given is statistical, the second systematic. Normalisation uncertainties of $10.1 \%$ are not included. 


\begin{tabular}{|c|c|c|c|c|}
\hline$Q^{2}\left[\mathrm{GeV}^{2}\right]$ & $\beta$ & $x_{\mathbb{P}}$ & $x_{\mathbb{P}} \sigma_{r}^{D(4)}\left[\mathrm{GeV}^{-2}\right]$ & $x_{\mathbb{P}} \sigma_{r}^{D(3)}$ \\
\hline 24.0 & 0.02 & 0.0220 & $0.0425 \pm 0.0088_{-0.0057}^{+0.0044}$ & $0.0306 \pm 0.0063_{-0.0042}^{+0.0033}$ \\
24.0 & 0.02 & 0.0450 & $0.0497 \pm 0.0090_{-0.0069}^{+0.0052}$ & $0.0341 \pm 0.0062_{-0.0048}^{+0.0038}$ \\
24.0 & 0.02 & 0.0800 & $0.0596 \pm 0.0128_{-0.0086}^{+0.0062}$ & $0.0394 \pm 0.0085_{-0.0058}^{+0.0043}$ \\
24.0 & 0.06 & 0.0100 & $0.0264 \pm 0.0061_{-0.0055}^{+0.0043}$ & $0.0200 \pm 0.0046_{-0.0042}^{+0.0033}$ \\
24.0 & 0.06 & 0.0220 & $0.0386 \pm 0.0070_{-0.0048}^{+0.0038}$ & $0.0276 \pm 0.0050_{-0.0034}^{+0.0028}$ \\
24.0 & 0.06 & 0.0450 & $0.0334 \pm 0.0075_{-0.0047}^{+0.0035}$ & $0.0231 \pm 0.0052_{-0.0033}^{+0.0026}$ \\
24.0 & 0.06 & 0.0800 & $0.0740 \pm 0.0187_{-0.0120}^{+0.0081}$ & $0.0480 \pm 0.0124_{-0.0079}^{+0.0055}$ \\
24.0 & 0.15 & 0.0040 & $0.0252 \pm 0.0068_{-0.0013}^{+0.0027}$ & $0.0190 \pm 0.0052_{-0.0010}^{+0.0021}$ \\
24.0 & 0.15 & 0.0100 & $0.0204 \pm 0.0038_{-0.0040}^{+0.0031}$ & $0.0152 \pm 0.0028_{-0.0030}^{+0.0024}$ \\
24.0 & 0.15 & 0.0220 & $0.0287 \pm 0.0051_{-0.0044}^{+0.0033}$ & $0.0206 \pm 0.0036_{-0.0032}^{+0.0024}$ \\
24.0 & 0.15 & 0.0450 & $0.0240 \pm 0.0064_{-0.0039}^{+0.0029}$ & $0.0164 \pm 0.0044_{-0.0027}^{+0.0021}$ \\
24.0 & 0.15 & 0.0800 & $0.0414 \pm 0.0181_{-0.0048}^{+0.0039}$ & $0.0274 \pm 0.0120_{-0.0033}^{+0.0028}$ \\
24.0 & 0.35 & 0.0040 & $0.0281 \pm 0.0072_{-0.0016}^{+0.0031}$ & $0.0214 \pm 0.0055_{-0.0013}^{+0.0025}$ \\
24.0 & 0.35 & 0.0100 & $0.0356 \pm 0.0074_{-0.0068}^{+0.0054}$ & $0.0266 \pm 0.0056_{-0.0051}^{+0.0004}$ \\
24.0 & 0.35 & 0.0220 & $0.0210 \pm 0.0060_{-0.0032}^{+0.0024}$ & $0.0153 \pm 0.0043_{-0.0024}^{+0.0008}$ \\
24.0 & 0.35 & 0.0450 & $0.0400 \pm 0.0183_{-0.0048}^{+0.0035}$ & $0.0274 \pm 0.0126_{-0.0034}^{+0.0026}$ \\
24.0 & 0.70 & 0.0011 & $0.0535 \pm 0.0125_{-0.0044}^{+0.0066}$ & $0.0410 \pm 0.0095_{-0.0035}^{+0.0052}$ \\
24.0 & 0.70 & 0.0040 & $0.0494 \pm 0.0106_{-0.0042}^{+0.0063}$ & $0.0378 \pm 0.0081_{-0.0033}^{+0.0049}$ \\
24.0 & 0.70 & 0.0100 & $0.0238 \pm 0.0071_{-0.0050}^{+0.0040}$ & $0.0177 \pm 0.0054_{-0.0038}^{+0.0033}$ \\
24.0 & 0.70 & 0.0220 & $0.0315 \pm 0.0103_{-0.0053}^{+0.0039}$ & $0.0231 \pm 0.0074_{-0.0039}^{+0.0029}$ \\
\hline
\end{tabular}

Table 8: The diffractive reduced cross sections, $x_{\mathbb{P}} \sigma_{r}^{D(4)}$ measured at $|t|=0.25 \mathrm{GeV}^{2}$, and $x_{\mathbb{P}} \sigma_{r}^{D(3)}$ integrated over $\left|t_{\min }\right|<|t|<1 \mathrm{GeV}^{2}$, measured at $Q^{2}=24 \mathrm{GeV}^{2}$ and various $\beta$ and $x_{\mathbb{P}}$ values. The fi rst uncertainty given is statistical, the second systematic. Normalisation uncertainties of $10.1 \%$ are not included. 